\title{
Mathematical Modelling and Analysis of Temperature Effects in MEMS
}

\author{
Von der Fakultät für Mathematik und Physik \\ der Gottfried Wilhelm Leibniz Universität Hannover \\ zur Erlangung des akademischen Grades \\ Doktor der Naturwissenschaften \\ Dr. rer. nat.
}

genehmigte Dissertation von

M.Sc. Tim Würth 
Referent: Prof. Dr. Joachim Escher

Koreferenten: Prof. Dr. Christoph Walker, Dr. Pierre Gosselet

Tag der Promotion: 27.09.2019 


\section{Contents}

$\begin{array}{lll}1 & \text { Abstract } & 5\end{array}$

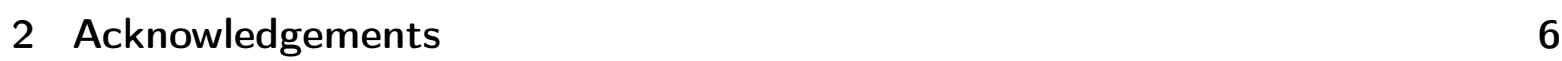

\begin{tabular}{lll}
\hline 3 & Introduction & 7
\end{tabular}

4 The full model $\quad 11$

4.1 The evolution of the membrane . . . . . . . . . . . . . . . . . . . . . 12

4.2 The electrostatic potential $\ldots \ldots \ldots \ldots$

4.3 The temperature . . . . . . . . . . . . . . . . . . 14

4.4 Transformation of the system . . . . . . . . . . . . . . . . . . 16

4.5 Examples for the temperature dependence $\ldots \ldots \ldots$. . . . . . . . . . . 18

5 The small aspect ratio limit 20

5.1 The system . . . . . . . . . . . . . . . . . . . . 20

5.2 Solution to the thermal and electrostatic problems . . . . . . . . . . . . . 21

5.3 Well-posedness of the deflection problem . . . . . . . . . . . . . . 22

6 Analysis of a 3d model 24

6.1 The model . . . . . . . . . . . . . . . . . . . . . . . . . . . . . . . 24

6.2 Assumptions on the temperature dependence . . . . . . . . . . . . . 27

6.3 Well-posedness of the electrostatic problem . . . . . . . . . . . . . . . . 28

6.4 Well-posedness of the deflection problem . . . . . . . . . . . . . . . 37

$\begin{array}{lll}7 & \text { Analysis of a } 2 \mathrm{~d} \text { model } & 40\end{array}$

7.1 The model . . . . . . . . . . . . . . . . . . . . . . . . . . . . . . . 40

7.2 Assumptions on the temperature dependence . . . . . . . . . . . . . . . 42

7.3 Well-posedness of the temperature problem . . . . . . . . . . . . . . . 43

7.4 Well-posedness of the deflection problem . . . . . . . . . . . . . . 47

8 Numerical analysis 56

8.1 Numerical setting . . . . . . . . . . . . . . . . . . . . 56

8.2 Results . . . . . . . . . . . . . . . . . . . . 57 
8.3 Influences of the parameters $\ldots \ldots \ldots \ldots \ldots$

8.3 .1 Temperature. . . . . . . . . . . . . . . . . . 60

8.3 .2 Shear modulus . . . . . . . . . . . . . . . . . 61

$8.3 .3 \quad$ No blowup . . . . . . . . . . . . . . . . . . . . . . . 61

8.4 Comparison between our model and the one without temperature effects 63

8.4 .1 M0 vs M2 . . . . . . . . . . . . . . . . . . . 63

$8.4 .2 \quad \mathrm{M} 0 \mathrm{vs} \mathrm{M} 2 \mathrm{f} \ldots \ldots \ldots \ldots \ldots$

$\begin{array}{lll}9 & \text { Bibliography } & 68\end{array}$

\begin{tabular}{ll}
\hline 10 Curriculum Vitae & 74
\end{tabular} 


\section{Abstract}

This thesis is concerned with the mathematical analysis of models for Micro-ElectroMechanical Systems (MEMS). These models arise in the form of coupled partial differential equations with a moving boundary. Although MEMS devices are often operated in non-isothermal environments, temperature is usually neglected in the mathematical investigations. Therefore the focus of our modelling is to incorporate temperature and the related material properties. We derive two models, both of which focus on different aspects of the underlying physics. Afterwards we prove local well-posedness in time and also global well-posedness under additional assumptions on the model's parameters. Lastly, we provide some numerical results which exemplify how temperature and the model's material constants change the qualitative behaviour of the system.

KEYWORDS: Micro-Electro-Mechanical Systems (MEMS), temperature, partial differential equations 


\section{Acknowledgements}

First and foremost I express my sincere gratitude to my supervisors Prof. Joachim Escher and Dr. Pierre Gosselet. I'm especially grateful for Prof. Escher's professional guidance, his availability whenever I had something to discuss and for proposing the research topic. Furthermore I thank Dr. Gosselet for the valuable advice and hints he gave me during the IRTG's meetings. I'm also greatly indebted to Prof. Christoph Walker for reviewing this thesis.

Further thanks goes to the members of the Institute of Applied Mathematics in particular to my colleagues Katerina Nik (M.Sc.) and Dr. Christina Lienstromberg for inspiring discussions and an excellent workplace environment. I also want to thank both Dr. Christina Lienstromberg and Dr. Christian Weißenfels for their support regarding the numerics.

Moreover I thank the IRTG 1627 for the financial support and the regular workshops. The latter allowed me to gain a broader perspective on my research. This was not only due to the talks held there but also due to the fruitful conversations with my IRTG colleagues for which I'm especially grateful.

Lastly, I want to thank my wife and my parents for their unconditional love and support. 


\section{Introduction}

\section{Introduction}

The technology of MEMS is concerned with microscopic devices that function by combining electrostatic with mechanical features. In his famous lecture from 1959 Richard Feynman discussed how very small machines could be used to solve a high variety of problems. However due to the difficult manufacturing a rapid development only started in the 1980s which in turn revolutionized numerous branches of industry [EGG10.

We now give some examples for such devices, for more details we refer to [Kaa09. Microsensors can be used to measure inertia, for example in airbags or to measure pressure for medical applications. Microactuators create a displacement by converting an electric signal into a mechanical output. Micropumps are used as a drug delivery system in medicine [NAMT08 or for a controlled transfer of fluids in chemical engineering Abi12.

Several of these devices actually rely on thermal effects: For example a thermal actuator creates motion by a thermal expansion that is due to resistive heating [PB02. Moreover a thermal-based microsensor can be used to monitor glucose and other metabolites |WSXL08|. Although other devices do not explicitly rely on temperature, they are nevertheless often operated in a non-isothermal environment. Possible examples are

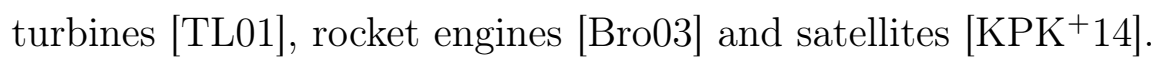

A key component (see Figure 1) of several MEMS devices consists of an elastic membrane and a fixed ground plate. A voltage is applied to the system and the resulting Coulomb force causes the membrane to deflect towards the ground plate. This change in gap size again impacts the electrostatics and thus the two effects are connected to each other. In order to design and optimize MEMS devices one has to gain a precise understanding of these underlying dynamics. It is therefore no surprise that several branches of science have advanced in this direction. We now turn to the recent mathematical investigations of MEMS.

A lot of mathematical research has been done focusing on the idealized MEMS-device with an electrical actuated membrane. Apart from the fact that this model is important for applications, there is also a purely mathematical reason for this interest. The device's 


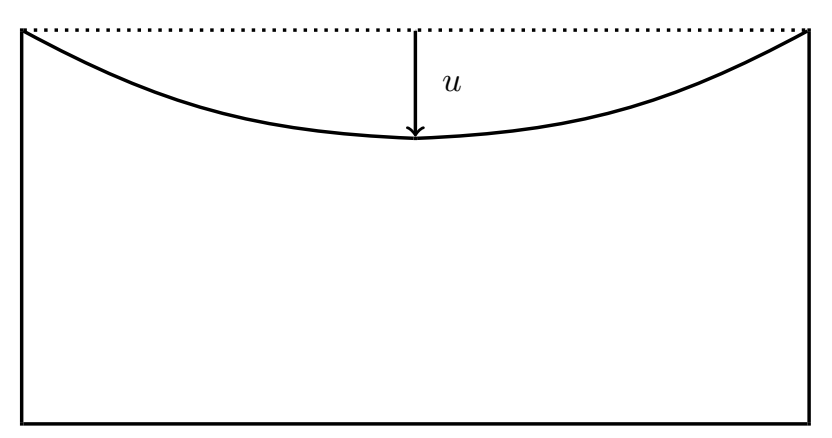

Figure 1: Idealized MEMS device in 2d with membrane $u$.

mechanics can only be fully understood if the model takes into account the fact that the device's boundary changes over time. The arising partial differential equations will therefore take the form of a moving boundary problem. These kinds of problems are of high interest in itself due to their inherently non-linear nature and their capability to describe natural phenomena accurately. Examples outside the world of MEMS devices include the Stefan problem, tumor growth models [BEL12 and the Muskat problem [EMW18]. The first mathematical analyses for MEMS devices use the additional assumption of a small aspect ratio (see for instance [LW14a, Hui11, Guo08, GG08, FMPS07, EG+ ${ }^{+}$08]). In the very recent contribution GZZ19 the authors consider an additional pressure term in the stationary case and examine positive solutions and singularties. Assuming a small aspect ratio makes it possible to decouple the electro and mechanical effects and thus consider a problem with a fixed boundary. The first results without this assumption were obtained in [ELW14 and in [LW13 for the stationary case. Building on this breakthrough several papers have been published that deal with different extensions of this model. For additional details we also refer to the surveys [LW17b, EL17 and the references therein.

In ELW15 the authors drop the usual assumption of small deformations and therefore consider a quasilinear equation for the membrane's deflection. The therein developed techniques will help us to handle a quasi-linearity which arises due to the temperature in our model.

The case of a general permittivity profile without assuming a small aspect ratio is investigated in [Lie15, Lie16] for small deformations and in [EL16 for non-small defor- 


\section{Introduction}

mations.

Moreover in LW14b, LW14c bending effects are included into the model and in |LW16 the domain of the ground plate is generalized from a simple interval to a convex and smooth $2 \mathrm{~d}$ domain. We will use the approach of the latter paper to prove well-posedness for a 3d model. Lastly, in [LW17a a constrained model is developed which allows further insights into the touchdown phenomena.

Some mathematicians in PDEs have already worked on incorporating temperature into models for MEMS. We want to emphasize that none of these authors considered the just described, well-established, moving boundary problem. However due to the proximity to our work we want to review this part of the literature as well. In AKS17 the authors consider the "V-shape" electro-thermal actuator. They show existence of a weak solution for their system and then carry out a numerical analysis. In |KHT16 the authors derive a model for the thermoelastic behaviour of a micro-beam resonator. Furthermore they solve the resulting equation analytically and show a good agreement to already available numerical data.

To the best of our knowledge no analytical work has been done that takes into account temperature effects on the dynamics of the above described idealized MEMS device. First steps in that direction were taken in [PB02, 4.4] and [PB02, 6.3.2]. However both approaches made use of significant simplifications. In [PB02, 4.4] the Joule heating of a cylinder is considered. The authors neglect a possible deflection of the cylinder's top and therefore only couple equations for electrostatic and thermal effects. In [PB02, 6.3.2] the authors ignore electrostatics and instead focus on the thermal-elastic behaviour. Additionally space variations of the temperature and damping of the membrane is neglected. Furthermore in both chapters the authors neither consider the question of well-posedness nor perform a numerical analysis. Instead they consider certain limits of the resulting equations and steady state solutions.

It is therefore our intention to fill this gap by incorporating temperature into the wellestablished moving boundary problem for the idealized MEMS device. We now outline the organisation of the thesis. 


\section{Introduction}

In section 4 we derive the full model in which electrostatic, thermal and mechanical effects are strongly coupled. The system will feature several parameters and general functions that depend on temperature. We then rescale the equations and discuss more concrete examples for the temperature dependence.

We start the analysis in section 5 by investigating the small aspect ratio limit. Then we consider two less simplified cases in sections 6 and 7. Both sections are organized in the same way: We first derive a simplification of the full model in $3 \mathrm{~d}$ and in $2 \mathrm{~d}$ respectively. In the $3 \mathrm{~d}$ case a higher focus is placed on how the temperature influences the electrostatic effects directly. In the $2 \mathrm{~d}$ case we instead focus on the temperature influence on the membrane's parameters. The resulting governing equation for the membrane will turn out to be quasilinear. We end both sections by proving local and global well-posedness of the derived problem under different assumptions.

Lastly, we complete this thesis with a numerical analysis of the $2 \mathrm{~d}$ case in section 8. We show how the different parameters influence the deflection of the membrane and in particular report significant differences between our model and the model M0 which neglects temperature effects. 


\section{The full model}

Our MEMS device is a cylinder with radius $a$ and height $h$, filled with a fluid. The fixed ground plate lies at $\widetilde{z}=-h$. We will use a tilde on several variables because we will rescale our equations later for convenience. The elastic membrane is attached at $\widetilde{z}=0$. Denoting the membrane's displacement with $\widetilde{u}$, the volume of the cylinder is given by

$$
V_{C}=\pi a^{2} h+\int_{\widetilde{E}} \widetilde{u}(\widetilde{x}, \widetilde{y}) d(\widetilde{x}, \widetilde{y})
$$

The ground plate and the region of the cylinder are denoted by

$$
\widetilde{E}:=\left\{(\widetilde{x}, \widetilde{y}) \mid \sqrt{\widetilde{x}^{2}+\widetilde{y}^{2}}<a\right\}
$$

and

$$
\widetilde{\Omega}(\widetilde{u}(\widetilde{t})):=\{(\widetilde{x}, \widetilde{y}, \widetilde{z}) \in \widetilde{E} \times(-h, \infty):-h<\widetilde{z}<u(\widetilde{x}, \widetilde{y}, \widetilde{t})\}
$$

respectively.

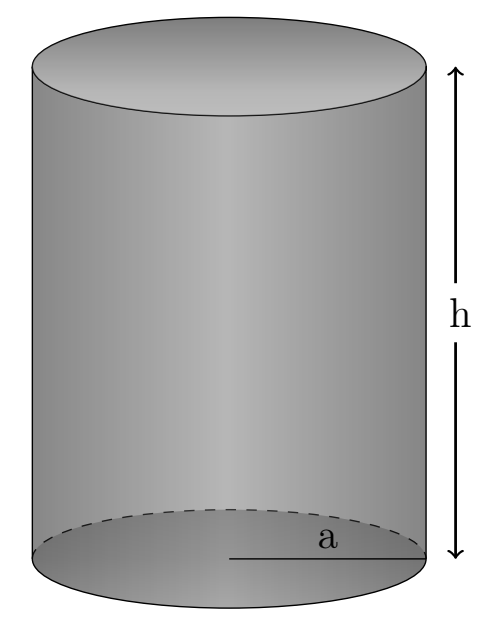

Figure 2: Domain $\widetilde{\Omega}(\widetilde{u}(\widetilde{t}))$ for $\widetilde{u}=0$.

In the following we assume that

$$
(\widetilde{x}, \widetilde{y}, \widetilde{z}) \in \widetilde{\Omega}(\widetilde{u}(\widetilde{t})), \widetilde{t} \in[0, \infty)
$$

holds if $\widetilde{x}, \widetilde{y}, \widetilde{z}$ and $\widetilde{t}$ are not specified otherwise. 


\subsection{The evolution of the membrane}

We formulate the equations governing the membrane's deflection which is modelled with a function $\widetilde{u}: \widetilde{E} \times[0, \infty) \rightarrow \mathbb{R}[$ PB02, page 192, page 236]:

$$
\begin{aligned}
& \rho_{m} d \frac{\partial^{2} \widetilde{u}}{\partial \widetilde{t^{2}}}(\widetilde{x}, \widetilde{y}, \widetilde{t})+a_{d} \frac{\partial \widetilde{u}}{\partial \widetilde{t}}(\widetilde{x}, \widetilde{y}, \widetilde{t}) \\
& -\mu(\widetilde{T}(\widetilde{x}, \widetilde{y}, \widetilde{u}(\widetilde{x}, \widetilde{y}, \widetilde{t}))) \widetilde{\nabla}_{\perp}^{2} \widetilde{u}(\widetilde{x}, \widetilde{y}, \widetilde{t})+D(\widetilde{T}(\widetilde{x}, \widetilde{y}, \widetilde{u}(\widetilde{x}, \widetilde{y}, \widetilde{t}))) \widetilde{\nabla}_{\perp}^{4} \widetilde{u}(\widetilde{x}, \widetilde{y}, \widetilde{t}) \\
& =-\frac{\epsilon_{1}(\widetilde{T})}{2}|\widetilde{\nabla} \widetilde{\psi}(\widetilde{x}, \widetilde{y}, \widetilde{u}(\widetilde{x}, \widetilde{y}, \widetilde{t}))|^{2}+P\left(\widetilde{T}(\widetilde{x}, \widetilde{y}, \widetilde{u}(\widetilde{x}, \widetilde{y}, \widetilde{t})), V_{C}(\widetilde{u}(\widetilde{x}, \widetilde{y}, \widetilde{t}))\right)
\end{aligned}
$$

In the membrane equation the electrostatic potential $\widetilde{\psi}$ and the temperature $\widetilde{T}$, which will be discussed shortly, are always evaluated on the membrane, that is $\widetilde{z}=\widetilde{u}(\widetilde{x}, \widetilde{y}, \widetilde{t})$. Therefore we will shorten the notation and write

$$
\rho_{m} d \frac{\partial^{2} \widetilde{u}}{\partial \widetilde{t}^{2}}+a_{d} \frac{\partial \widetilde{u}}{\partial \widetilde{t}}-\mu(\widetilde{T}) \widetilde{\nabla}_{\perp}^{2} \widetilde{u}+D(\widetilde{T}) \widetilde{\nabla}_{\perp}^{4} \widetilde{u}=-\frac{\epsilon_{1}(\widetilde{T})}{2}|\widetilde{\nabla} \widetilde{\psi}|^{2}+P\left(\widetilde{T}, V_{C}\right)
$$

instead of 4.2 whenever it is convenient.

We use the notation $\widetilde{\nabla}_{\perp}^{2}:=\partial_{\widetilde{x}}^{2}+\partial_{\widetilde{y}}^{2}$. Here $d$ is the membrane's thickness, $\rho_{m}$ the density of the membrane's material, $a_{d}$ the damping constant, $\mu(\widetilde{T})$ the tension in the membrane, $D(\widetilde{T})$ its flexural rigidity and $\epsilon_{1}(\widetilde{T})$ the permittivity. Furthermore $P$ is the pressure and $\widetilde{T}$ the temperature. The right-hand side of the latter equation captures the fact that the membrane's movement is dependent on both the electrostatic potential and the temperature within the device. The voltage difference pulls the membrane to the ground plate whereas the pressure of the fluid, which increases with temperature, pushes the membrane upwards.

At the edge of the cylinder the membrane is clamped and it is assumed to start in position $u_{0}$. Therefore we have the boundary and initial conditions

$$
\widetilde{u}(\widetilde{x}, \widetilde{y}, \widetilde{t})=\partial_{r} \widetilde{u}(\widetilde{x}, \widetilde{y}, \widetilde{t})=0, \text { for }(\widetilde{x}, \widetilde{y}) \in \partial \widetilde{E}, \widetilde{t} \in[0, \infty)
$$

and

$$
\widetilde{u}(\widetilde{x}, \widetilde{y}, 0)=u_{0}(\widetilde{x}, \widetilde{y}), \quad \partial_{r} \widetilde{u}(\widetilde{x}, \widetilde{y}, 0)=u_{1}(\widetilde{x}, \widetilde{y}) \text { for }(\widetilde{x}, \widetilde{y}) \in \widetilde{E}
$$



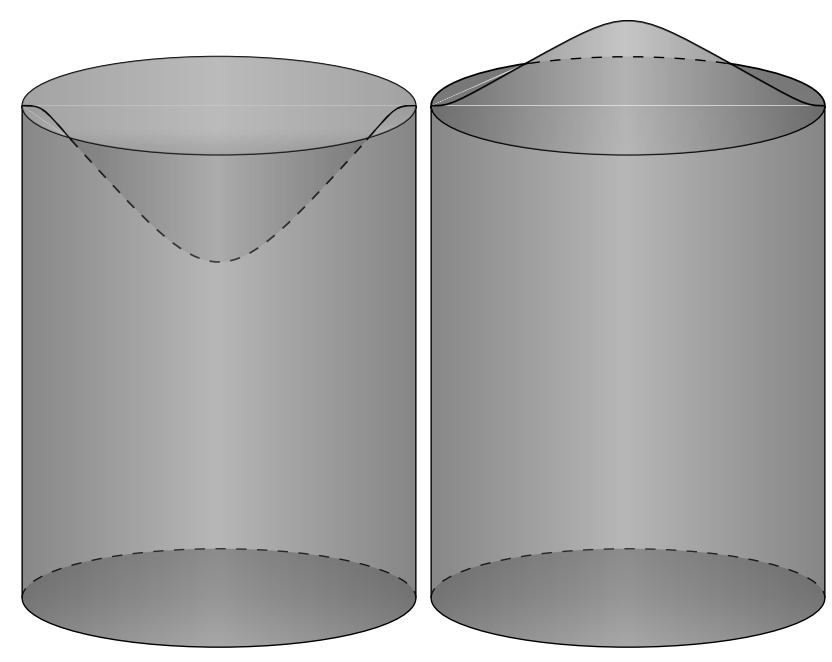

Figure 3: Domain $\widetilde{\Omega}(\widetilde{u}(\widetilde{t}))$ for a membrane with a negative and a positive deflection.

where $u_{0}$ and $u_{1}$ are given initial data.

\subsection{The electrostatic potential}

We state the governing equations for the electrostatic potential within the cylinder. A tilde on differential operators denotes derivatives with respect to variables with a tilde. $\sigma(\widetilde{T}): \mathbb{R} \rightarrow \mathbb{R}$ is the conductivity of the fluid in the cylinder which will depend on the temperature. We model the electrostatic potential with the equation

$$
\widetilde{\nabla} \cdot(\sigma(\widetilde{T}) \widetilde{\nabla} \widetilde{\psi})(\widetilde{x}, \widetilde{y}, \widetilde{z})=0
$$

A derivation of the latter can be found in $\mathrm{PB} 02$, page 104]. By defining $\sigma(\widetilde{T})$ as a scalar only depending on temperature and not as a tensor depending on the position inside the cylinder we assumed that the fluid is homogeneous and isotropic. The fixed plate at $\widetilde{z}=-h$ is grounded and the potential in the amount of $V$ is applied to the membrane. Also we assume that there is no current through the lateral boundary of the cylinder. Denoting the derivative in the outward radial direction by $\partial_{r}$ this imposes the boundary conditions [PB02, page 104]:

$$
\widetilde{\psi}(\widetilde{x}, \widetilde{y},-h)=0, \quad \widetilde{\psi}(\widetilde{x}, \widetilde{y}, \widetilde{u}(\widetilde{x}, \widetilde{y}))=V, \quad \partial_{r} \widetilde{\psi}=\left.0\right|_{r=a}
$$




\subsection{The temperature}

Now we turn to the thermal problem: We derive a diffusion advection equation for the temperature $\widetilde{T}$ similar to $\operatorname{Sin} 13, \mathrm{CA} 86$ : Here $\rho_{f}$ is the density of the fluid, $c$ is the specific heat capacity. We write $\rho_{f}=\frac{m}{V_{C}}$ where $m$ is the mass of the whole fluid. This makes the density time-dependent because $V_{C}$ depends on the membrane's position. We do however assume that density variations in space are neglectable. Thermal energy is then given by $\rho_{f} c \widetilde{T}$. The continuity equation in differential form which relates the change of the thermal energy to the heat flux $j$ and to the source term $R$ is given by:

$$
\frac{\partial \rho_{f} c \widetilde{T}}{\partial \widetilde{t}}+\nabla \cdot j=R
$$

Our source term captures two effects. Firstly the electric energy which is converted to thermal energy $\mathrm{PB} 02$, page 105] and secondly the pressure-volume work (or volumechange work) [PB02, page 189]. Pressure-volume work is relevant in our case because if the cylinders volume decreases, the heat energy inside the cylinder increases 1 Consequently the source term is of the form

$$
R=\sigma(\widetilde{T})|\widetilde{\nabla} \widetilde{\psi}|^{2}-P\left(\widetilde{T}, V_{C}\right) \frac{d V_{C}}{d \widetilde{t}}
$$

Here we decided not to include the heat energy generated by elastic energy. This is reasonable because this amount of heat is outweighed by Joule heating [PB02, page 38]. We consider two different types of flux: The diffusive flux $j_{\text {diff }}$ which corresponds to heat conduction in our setting can be approximated by Fourier's first law:

$$
j_{\text {diff }}=-k \widetilde{\nabla} \widetilde{T}
$$

The thermal conductivity $k$ is assumed to be constant. The advective flux $j_{\text {adv }}$ gives the bulk motion of our fluid in the cylinder in direction $v$ :

$$
j_{\mathrm{adv}}=v \widetilde{T} .
$$

\footnotetext{
${ }^{1}$ Here we used the in our case reasonable assumption that the described process is reversible. PB02, page 189] Also just like with density we assumed that space variations for the pressure-volume work are neglectable.
} 
Now we plug the flux $\left(j=j_{\text {adv }}+j_{\text {diff }}\right)$ and the source term into the continuity equation and assume $c$ to be constant in order to obtain

$$
c \frac{\partial \rho_{f} \widetilde{T}}{\partial \widetilde{t}}=k \widetilde{\triangle} \widetilde{T}-\widetilde{\nabla} \cdot(v \widetilde{T})+\sigma(\widetilde{T})|\widetilde{\nabla} \widetilde{\psi}|^{2}-P\left(T, V_{C}\right) \frac{d V_{C}}{d \widetilde{t}} .
$$

The velocity $v$ in the advection term $-\widetilde{\nabla} \cdot(v \widetilde{T})$ is hard to handle if the device is turned or moved. This is why we want to ignore it. This means we consider the case in which conduction dominates advection. Fortunately this approximation is very reasonable for our case: Since we are dealing with a microsystem we can assume a small Reynolds number $R e$ [PB02 which in turn yields a small Rayleigh number ${ }^{2}$ GL02. Furthermore for a laminar flow (again a reasonable assumption in the regime of a small Reynolds number [PB02, page 307]) the length of the boundary layer is approximately $\frac{r}{\sqrt{R e}}[\mathrm{~PB} 02$, page 305]. This makes the boundary layer's thickness larger than our system which in turn results in slow velocities. Also diffusion is relatively fast in small length scales $\mathrm{PB} 02$, page 83]. Therefore we reduce our initial equation to:

$$
c \frac{\partial \rho_{f} \widetilde{T}}{\partial \widetilde{t}}=k \widetilde{\triangle} \widetilde{T}+\sigma(\widetilde{T})|\widetilde{\nabla} \widetilde{\psi}|^{2}-P\left(\widetilde{T}, V_{C}\right) \frac{d V_{C}}{d \widetilde{t}}
$$

In our setting this can be rewritten as:

$$
\frac{c m}{V_{C}} \frac{\partial \widetilde{T}}{\partial \widetilde{t}}=k \widetilde{\triangle} \widetilde{T}+\sigma(\widetilde{T})|\widetilde{\nabla} \widetilde{\psi}|^{2}-c \widetilde{T} m \frac{\partial\left(V_{C}^{-1}\right)}{\partial \widetilde{t}}-P\left(\widetilde{T}, V_{C}\right) \frac{d V_{C}}{d \widetilde{t}}
$$

We assume that the cylinder is at ambient temperature $T_{A}$ at time $\tilde{t}=0$ and that the membrane is insulated. We allow for heat loss through the lateral sides of the cylinder's boundary. The heat loss is modelled by the Newton cooling condition with heat transfer coefficient $h_{n c}$. This gives us the boundary conditions:

$$
\widetilde{T}(\widetilde{x}, \widetilde{y}, \widetilde{z}, 0)=T_{0}, \quad \frac{\partial \widetilde{T}}{\partial n}=\left.0\right|_{\widetilde{z}=\widetilde{u}(\widetilde{x}, \widetilde{y}, \widetilde{z}, \widetilde{t})}, \quad k \frac{\partial \widetilde{T}}{\partial \widetilde{r}}=-\left.h_{n c}\left(\widetilde{T}-T_{0}\right)\right|_{r=a}
$$

\footnotetext{
${ }^{2}$ This number gives the ratio between the gravitional and viscous forces.
} 
Here $n$ denotes the unit normal at the membrane. We also assume that the cylinder is heated from below. This is modelled by a given function $T_{h}: \widetilde{E} \times[0, \infty)$ :

$$
\widetilde{T}(\widetilde{x}, \widetilde{y},-h, \widetilde{t})=\widetilde{T_{h}}(\widetilde{x}, \widetilde{y}, \widetilde{t})
$$

\subsection{Transformation of the system}

Now we introduce dimensionless variables

$$
\begin{gathered}
x:=\frac{\widetilde{x}}{a}, \quad y:=\frac{\widetilde{y}}{a}, \quad z:=\frac{\widetilde{z}}{h}, \quad t:=\frac{\widetilde{t}}{a_{d} a^{2}}, \\
u:=\frac{\widetilde{u}}{h}, \quad \psi:=\frac{\widetilde{\psi}}{V}, \quad T:=\widetilde{T}, \quad T_{h}(x, y, t):=\widetilde{T_{h}}(\widetilde{x}, \widetilde{y}, \widetilde{t}) .
\end{gathered}
$$

We further define domains

$$
E:=\left\{(x, y) \mid \sqrt{x^{2}+y^{2}}<1\right\}
$$

and

$$
\Omega(u(t)):=\{(x, y, z) \in E \times(-1, \infty):-1<z<u(x, y, t)\}
$$

In the following we assume that

$$
(x, y, z) \in \Omega(u(t))
$$

holds if not specified otherwise. We now use these variables and multiply the governing equation 4.5 for the potential $\psi$ by $\frac{h^{2}}{V}$, the governing equation 4.13 for the temperature $T$ by $\frac{h^{2}}{k}$ and the governing equation 4.2 for the membrane's displacement by $\frac{a^{2}}{h}$. Our equations in dimensionless form are

$$
\frac{h^{2}}{a^{2}} \nabla_{\perp} \cdot\left(\sigma(T) \nabla_{\perp} \psi\right)+\frac{\partial}{\partial z}\left(\sigma(T) \frac{\partial}{\partial z} \psi\right)=0
$$




$$
\begin{aligned}
& \frac{c h^{2} m}{a_{d} k a^{2} V_{C}} \frac{\partial T}{\partial t}=\left(\frac{h^{2}}{a^{2}} \nabla_{\perp}^{2} T+\frac{\partial^{2}}{\partial^{2} z} T\right) \\
& \left.+\sigma(T) \frac{V^{2}}{k}\left(\frac{h^{2}}{a^{2}}\left|\nabla_{\perp} \psi\right|^{2}+\left(\frac{\partial \psi}{\partial z}\right)^{2}\right)-\frac{c h^{2} m}{a_{d} k a^{2}} T \frac{\partial\left(V_{C}^{-1}\right)}{\partial t}\right)-\frac{h^{2}}{k a_{d} a^{2}} P\left(T, V_{C}\right) \frac{d V_{C}}{d t}
\end{aligned}
$$

and

$$
\frac{\partial u}{\partial t}-\mu(T) \nabla_{\perp}^{2} u+\frac{D}{a^{2}} \nabla_{\perp}^{4} u=-\frac{\epsilon_{1}(T) V^{2} a^{2}}{2 h^{3}}\left(\frac{h^{2}}{a^{2}}\left|\nabla_{\perp} \psi\right|^{2}+\left(\frac{\partial \psi}{\partial z}\right)^{2}\right)+\frac{a^{2}}{h} P\left(T, V_{C}\right)
$$

We also simplified the equation for the membrane's displacement further by assuming that the thickness of the membrane $d$ is small so we can drop the second time derivative. This is a standard simplification which is used for example in PB02, ELW14, GPW05, EGG10]. This assumption is valid for micropumps and other MEMS devices that are described in the mentioned references. The boundary and initial conditions now yield:

$$
\begin{gathered}
\psi(x, y,-1)=0, \quad \psi(x, y, u(x, y, t))=1, \quad \frac{\partial \psi}{\partial r}=\left.0\right|_{r=1}, \\
T(x, y, z, 0)=T_{0}, \quad \frac{\partial T}{\partial n}=\left.0\right|_{z=u(x, y, t)}, \quad \frac{k}{a} \frac{\partial T}{\partial r}=-\left.h_{n c}\left(T-T_{0}\right)\right|_{r=1}, \\
T(x, y,-1, t)=T_{h}(x, y, t), \\
u(x, y, t)=0, \text { for }(x, y) \in \partial E \text { and } u(x, y, 0)=u_{0} \text { for }(x, y) \in E \\
u(x, y, t)=\partial_{r} u(x, y, t)=0 \quad(x, y) \in \partial E
\end{gathered}
$$

With the definitions for the aspect ratio $\epsilon$ and the 'tuning parameters' $\lambda_{i}$ :

$$
\epsilon:=\frac{h}{a}, \quad \lambda_{1}:=\frac{V^{2} a^{2}}{2 h^{3}}, \lambda_{2}:=\frac{a^{2}}{h}, \quad \lambda_{3}:=\frac{c m}{a_{d} k}, \lambda_{4}=\frac{V^{2}}{k}, \quad \lambda_{5}=\frac{1}{k a_{d}}, \quad \lambda_{6}=\frac{1}{a^{2}} .
$$

We now state our governing equations one last time by also replacing the terms containing $V_{C}$, which is given by (4.1), by terms containing $u$ :

$$
\epsilon^{2}\left(\sigma(T) \partial_{x}^{2} \psi+\sigma(T) \partial_{y}^{2} \psi+\partial_{x} \sigma(T) \partial_{x} \psi+\partial_{y} \sigma(T) \partial_{y} \psi\right)+\sigma(T) \partial_{z}^{2} \psi+\partial_{z} \sigma(T) \partial_{z} \psi=0
$$




$$
\begin{gathered}
\frac{\lambda_{3} \epsilon^{2}}{\pi a^{2} h+\int_{E} u d x d y} \partial_{t} T=\epsilon^{2} \nabla_{\perp}^{2} T+\partial_{z}^{2} T+\sigma(T) \lambda_{4}\left[\epsilon^{2}\left|\nabla_{\perp} \psi\right|^{2}+\left(\partial_{z} \psi\right)^{2}\right] \\
+\frac{\lambda_{3} \epsilon^{2} T \int_{E} \partial_{t} u d x d y}{\left(\pi a^{2} h+\int_{E} u d x d y\right)^{2}}-\epsilon^{2} \lambda_{5} P(T, u) \int_{E} \partial_{t} u d x d y, \\
\partial_{t} u-\mu(T) \nabla_{\perp}^{2} u+D(T) \lambda_{6} \nabla_{\perp}^{4} u=-\epsilon_{1}(T) \lambda_{1}\left[\epsilon^{2}\left|\nabla_{\perp} \psi\right|^{2}+\left(\partial_{z} \psi\right)^{2}\right]+\lambda_{2} P(T, u) .
\end{gathered}
$$

\subsection{Examples for the temperature dependence}

A variety of different materials are used in MEMS. The specific form for the electric conductivity $\sigma(T)$, permittivity of the membrane $\epsilon_{1}(T)$, pressure $P(T, u)$ and shear modulus $\mu(T)$ strongly depend on the situation ${ }^{3}$ and in particular the material in question. Furthermore these properties have to be validated in experiments. Therefore we do not chose specific forms for these four and instead let them be arbitrary functions satisfying hypotheses appropriate for our mathematical analysis. Nevertheless we want to give some examples how they could look like:

For Sylgard 184 the shear modulus (second Lamé constant) $\mu$ depends linearly on temperature:

$$
\mu(T)=\mu_{0} T+\mu_{1}
$$

The temperature dependence (for low temperatures) of the permittivity $\epsilon_{1}(T)$ of the polymer PMMA can be approximated linearly as well [SKR $\left.{ }^{+} 01\right]$.

For the pressure in a gas we can use the ideal gas law. $N$ is the number of moles in the gas and $R$ is the ideal gas constant. The pressure $P$ is then given by

$$
P=\frac{N R T}{V_{C}}
$$

For liquids there are other equations of state (EOS), for example the Peng-Robinson

\footnotetext{
${ }^{3}$ By situation we mean for example the temperature regime or the size of the cylinder.

${ }^{4}$ This material is used for micropumps. Then we have $\mu_{0}=0,0032$ and $\mu_{1}=0,373$ [JMTT14, Table 4].
} 
EOS (which can also be used for gas):

$$
P=\frac{R T}{V_{m}-b}-\frac{a \alpha}{V_{m}^{2}+2 b V_{m}-b^{2}}
$$

For the interpretation of the real constants $R, V_{m}, b, \alpha$ and $a$ we refer the interested reader to $\mathrm{PR} 76$.

The electric conductivity of a liquid can be approximated as an exponential function (see e.g.: [BR98, Chapter 4 and 5], $\left.\mathrm{VGP}^{+} 06\right]$ ):

$$
\sigma(T)=\sigma_{\infty} \exp \left(\frac{E_{\alpha}}{k_{B} T}\right)
$$

Where $\sigma_{\infty}$ denotes the maximum electrical conductivity, $k_{B}$ the Boltzmann constant and $E_{a}$ the activation energy.

Gases however have neglectable electric conductivity. 


\section{The small aspect ratio limit}

This section should be viewed as a prototype for sections 6 and 7 because we will briefly go through the same steps but in a highly simplified setting. We consider the case of a small aspect ratio. To be precise, we make the following simplifications: We let $\epsilon \rightarrow 0$, the dimension is reduced to two, we impose Dirichlet boundary conditions for both temperature and electrostatic potential and we let $\sigma(T):=\sigma$ be independent of temperature.

\subsection{The system}

We define

$$
I:=(-1,1)
$$

and

$$
\Omega(u(t)):=\{(x, z) \in I \times(-1, \infty):-1<z<u(x, t)\} .
$$

The electrostatic potential $\psi$ is governed by

$$
\sigma \partial_{z}^{2} \psi(x, z)=0, \quad(x, z) \in \Omega(u(t)), t>0
$$

together with the boundary conditions

$$
\psi(t, x, z)=\frac{1+z}{1+u(t, x)},(x, z) \in \partial \Omega(u(t)), t>0 .
$$

The temperature $T$ is governed by

$$
\partial_{z}^{2} T(x, z)=-\sigma \lambda_{4}\left(\partial_{z} \psi\right)^{2} \quad(x, z) \in \Omega(u(t)), \quad t>0,
$$

with the boundary conditions

$$
T(t, x,-1)=T_{0}, \quad T(t, x, u(x, t))=T_{1}, \quad t>0, \quad x \in I,
$$


for $T_{0}>T_{1}>0$. Let $t>0$ and $x \in I$, the membrane's deflection $u$ is governed by

$$
\partial_{t} u(t, x)-\mu(T(x, u(t, x))) \partial_{x}^{2} u(t, x)=-\frac{\lambda_{1} \epsilon_{1}(T(x, u(t, x)))}{(1+u(t, x))^{2}}+\lambda_{2} P(T(x, u(t, x)), u(t, x))
$$

with the boundary conditions

$$
u( \pm 1, t)=0, t>0 \text { and } u(x, 0)=u_{0}(x) \text { for } x \in I .
$$

We can now solve explicitly for both the electrostatic potential and the temperature.

\subsection{Solution to the thermal and electrostatic problems}

The solution to the electrostatic problem may be written as

$$
\psi(t, x, z)=\frac{1+z}{1+u(t, x)}, \quad t>0, \quad(x, z) \in \Omega(u(t)) .
$$

Inserting this expression in the temperature equation, it may be rewritten as

$$
\partial_{z}^{2} T(t, x, z)=-\frac{\sigma \lambda_{4}}{(1+u(t, x))^{2}}, \quad t>0, \quad(x, z) \in \Omega(u(t))
$$

Since the RHS of this equation is independent of $z$, we can solve it explicitly by integrating. The solution to the temperature problem may be written as

$$
T(t, x, z)=A z^{2}+B z+C
$$

with

$$
\begin{gathered}
A:=A(u(t, x)):=-\frac{\sigma \lambda_{4}}{2(1+u(t, x))^{2}}, \\
B:=B(u(t, x)):=\frac{T_{1}-T_{0}}{1+u(t, x)}+\frac{\sigma \lambda_{4}(u(t, x)-1)}{2(1+u(t, x))^{2}}
\end{gathered}
$$

and

$$
C:=C(u(t, x)):=\frac{T_{1}-T_{0}}{1+u(t, x)}+T_{0}+\frac{\sigma \lambda_{4} u(t, x)}{2(1+u(t, x))^{2}}
$$




\subsection{Well-posedness of the deflection problem}

For the reader's convenience we present the the full system for this model again:

$$
\begin{gathered}
T_{u}(t, x, z)=A(u(t, x)) z^{2}+B(u(t, x)) z+C(u(t, x)), \\
\psi_{u}(t, x, z)=\frac{1+z}{1+u(t, x)}, \\
u_{t}-\mu\left(T_{1}\right) \partial_{x}^{2} u=-\frac{\epsilon_{1}\left(T_{1}\right) \lambda_{1}}{(1+u)^{2}}+\lambda_{2} P\left(T_{1}, u\right), \\
u( \pm 1, \cdot)=0, \quad \text { and } u(\cdot, 0)=u_{0} .
\end{gathered}
$$

Let $p \in(1, \infty)$. We define the spaces:

$$
W_{p, \mathcal{B}}^{2 \alpha}(I)=\left\{\begin{array}{c}
W_{p}^{2 \alpha}(I), 2 \alpha \in[0,1 / p), \\
\left\{u \in W_{p}^{2 \alpha}(I) ; u( \pm 1)=0\right\}, 2 \alpha \in(1 / p, 2] .
\end{array}\right.
$$

Since

$$
T(t, x, u(x, t))=T_{1}, x \in I, t>0
$$

we can uncouple the temperature from the membrane's deflection and write $P(u, T)=$ $P(u), \epsilon(T)=\epsilon_{1}$, and $\mu(T)=\mu$. Thus we can simplify the initial value problem for the membrane's equation:

$$
\begin{aligned}
& u_{t}-\mu \partial_{x}^{2} u=g(u), \quad t>0, \quad x \in I, \\
& u(0, x)=u_{0}(x), \quad x \in I \\
& u(t, \pm 1)=0, \quad t>0
\end{aligned}
$$

with

$$
g(u):=-\frac{\epsilon_{1} \lambda_{1}}{(1+u)^{2}}+\lambda_{2} P(u)
$$

We have the following result:

Theorem 5.1. Let $p \in(1, \infty)$ and $u \rightarrow P(u)$ be globally Lipschitz. For each $u_{0} \in$ $W_{p, \mathcal{B}}^{2}(I)$ with $u_{0}>-1$ there is a positive existence time $t_{1}>0$ and a unique solution 
$u(t, x)$ to 5.10$)$ satisfying

$$
u \in C\left(\left[0, t_{1}\right), W_{p, \mathcal{B}}^{2}\right) \cap C^{1}\left(\left(0, t_{1}\right), L_{p}(I)\right) .
$$

This theorem is due to a standard argument which we outline here: The operator

$$
A u:=\mu \partial_{x}^{2} u, u \in W_{p, \mathcal{B}}^{2}
$$

is uniformly strongly elliptic (see e.g. Ama93, Example 4.3a]). Since we have simple Dirichlet boundary conditions the boundary value problem $(A, \mathcal{B})$ is normally elliptic Ama93, Remark 4.3c] and therefore

$$
A \in \mathcal{H}\left(W_{p, \mathbb{B}}^{2}(I), L_{p}(I)\right)
$$

follows from Ama93, Theorem 4.1]. Denote by $S(t)$ the semigroup which is generated by $A$. The exponential decay

$$
\|S(t)\|_{\mathcal{L}\left(L_{p}(I)\right)} \leq M e^{-\nu t}, M \geq 1, \nu>0
$$

can be proven by applying $[$ Paz12, Chapter 4.4, Theorem 4.3]. Finally, the result follows for example by Ama93, Remark 12.2b]. 


\section{Analysis of a $3 d$ model}

In this section we will propose and analyse a $3 \mathrm{~d}$ version of the full model.

\subsection{The model}

Apart from staying in three dimensions we also keep the 4th order term in the equation which governs the displacement of the membrane.

However we do make the following simplifications: Instead of letting the temperature be modelled by a PDE we assume that a temperature profile $\mathcal{T} \in B U C^{1}(\Omega)$ is given. Furthermore we let $\mu(\mathcal{T})=\mu>0$ and $D(\mathcal{T})=D>0$ be fixed. That means we assume that temperature induced changes of the shear modulus and the flexural rigidity are neglectable. Lastly we consider Dirichlet boundary conditions for $\phi$ as for example in [LW16, ELW15, ELW14.

Before we state the resulting model, we first transform the moving boundary to a fixed domain. We fix the time $t$ and let $q \geq 2, u \in W_{q}^{2}(E)$ and $\mathcal{T} \in B U C^{1}(\Omega)$ be given. We consider the problem for the electrostatic potential:

$$
\begin{gathered}
\epsilon^{2}\left(\sigma(\mathcal{T}) \partial_{x}^{2} \psi_{u}+\sigma(\mathcal{T}) \partial_{y}^{2} \psi_{u}+\partial_{x} \sigma(\mathcal{T}) \partial_{x} \psi_{u}+\partial_{y} \sigma(\mathcal{T}) \partial_{y} \psi_{u}\right) \\
+\sigma(\mathcal{T}) \partial_{z}^{2} \psi_{u}+\partial_{z} \sigma(\mathcal{T}) \partial_{z} \psi_{u}=0 \quad \text { in } \Omega(u) \\
\psi_{u}(x, y, z)=\frac{1+z}{1+u(x, y)}, \quad(x, y, z) \in \partial \Omega(u) .
\end{gathered}
$$

The fixed domain is given by

$$
\Omega:=E \times(0,1)
$$

with

$$
E:=\left\{(x, y) \mid \sqrt{x^{2}+y^{2}}<1\right\}
$$

We use the diffeomorphism $D_{u}: \overline{\Omega(u)} \rightarrow \bar{\Omega}$, with

$$
D_{u}(x, y, z):=\left(x, y, \frac{1+z}{1+u(x, y)}\right), \quad(x, y, z) \in \overline{\Omega(u)}
$$


The inverse is

$$
D_{u}^{-1}(x, y, \eta):=(x, y,(1+u(x, y)) \eta-1), \quad(x, y, \eta) \in \bar{\Omega}
$$

After the corresponding coordinate transformation we get the following operator:

$$
\begin{aligned}
& \mathfrak{L}_{(u, T)} w:= \\
& \sigma(T)\left(\epsilon^{2} \partial_{x}^{2} w+\epsilon^{2} \partial_{y}^{2} w-2 \epsilon^{2} \eta \frac{\partial_{x} u}{1+u} \partial_{x} \partial_{\eta} w-2 \epsilon^{2} \eta \frac{\partial_{y} u}{1+u} \partial_{y} \partial_{\eta} w\right. \\
& \left.+\frac{1+\epsilon^{2} \eta^{2}|\nabla u|^{2}}{(1+u)^{2}} \partial_{\eta}^{2} w\right)+\left(\epsilon ^ { 2 } \eta \left(\sigma(T)\left[2 \frac{|\nabla u|^{2}}{(1+u)^{2}}-\frac{\Delta u}{1+u}\right]+\partial \sigma(T)\left[-\frac{\partial_{x} u}{1+u}\left(\partial_{x} T\right.\right.\right.\right. \\
& \left.\left.\left.\left.-\frac{\eta \partial_{x} u}{1+u} \partial_{\eta} T\right)-\frac{\partial_{y} u}{1+u}\left(\partial_{y} T-\frac{\eta \partial_{y} u}{1+u} \partial_{\eta} T\right)\right]\right)+\frac{\partial \sigma(T) \partial_{\eta} T}{(1+u)^{2}}\right) \partial_{\eta} w \\
& +\epsilon^{2} \partial \sigma(T)\left(\partial_{x} T-\partial_{\eta} T \frac{\eta \partial_{x} u}{1+u}\right) \partial_{x} w+\epsilon^{2} \partial \sigma(T)\left(\partial_{y} T-\partial_{\eta} T \frac{\eta \partial_{y} u}{1+u}\right) \partial_{y} w .
\end{aligned}
$$

For later purposes we split this operator as

$$
\mathfrak{L}_{(u, T)}=\sigma(T) \mathfrak{L}_{(u, T)}^{1}+\mathfrak{L}_{(u, T)}^{2}
$$

with

$$
\begin{aligned}
& \mathfrak{L}_{(u, T)}^{1} w:=\epsilon^{2} \partial_{x}^{2} w+\epsilon^{2} \partial_{y}^{2} w-2 \epsilon^{2} \eta \frac{\partial_{x} u}{1+u} \partial_{x} \partial_{\eta} w-2 \epsilon^{2} \eta \frac{\partial_{y} u}{1+u} \partial_{y} \partial_{\eta} w \\
& +\frac{1+\epsilon^{2} \eta^{2}|\nabla u|^{2}}{(1+u)^{2}} \partial_{\eta}^{2} w+\epsilon^{2} \eta\left[2 \frac{|\nabla u|^{2}}{(1+u)^{2}}-\frac{\Delta u}{1+u}\right] \partial_{\eta} w
\end{aligned}
$$

and

$$
\begin{aligned}
& \mathfrak{L}_{(u, T)}^{2} w:= \\
& \left(\epsilon^{2} \eta \partial \sigma(T)\left[-\frac{\partial_{x} u}{1+u}\left(\partial_{x} T-\frac{\eta \partial_{x} u}{1+u} \partial_{\eta} T\right)-\frac{\partial_{y} u}{1+u}\left(\partial_{y} T-\frac{\eta \partial_{y} u}{1+u} \partial_{\eta} T\right)\right]+\frac{\partial \sigma(T) \partial_{\eta} T}{(1+u)^{2}}\right) \partial_{\eta} w \\
& +\epsilon^{2} \partial \sigma(T)\left(\partial_{x} T-\partial_{\eta} T \frac{\eta \partial_{x} u}{1+u}\right) \partial_{x} w+\epsilon^{2} \partial \sigma(T)\left(\partial_{y} T-\partial_{\eta} T \frac{\eta \partial_{y} u}{1+u}\right) \partial_{y} w
\end{aligned}
$$


This is convenient because $\mathfrak{L}_{(u, T)}^{1}$ is exactly the operator used in [LW16, p. 398]. With the definitions

$$
\phi_{u}:=\psi_{u} \circ D_{u}^{-1}
$$

and

$$
T:=\mathcal{T} \circ D_{u}^{-1}
$$

the electrostatic potential's problem $(6.1)$ and $(6.2)$ can now be written as:

$$
\begin{gathered}
\mathfrak{L}_{(u, T)} \phi_{u}(x, y, \eta)=0, \quad(x, y, \eta) \in \Omega, \\
\phi_{u}(x, y, \eta)=\eta, \quad(x, y, \eta) \in \partial \Omega .
\end{gathered}
$$

For $q \geq 3$ we have $T \in W_{\infty}^{1}(\Omega)$. The problem for the membrane's displacement in the new coordinates is

$$
\begin{gathered}
u(x, y, t)=\partial_{r} u(x, y, t)=0 \quad(x, y) \in \partial E, \\
\partial_{t} u-\mu \nabla_{\perp}^{2} u+D \lambda_{6} \nabla_{\perp}^{4} u=g(u),
\end{gathered}
$$

with

$$
g(v):=-\epsilon_{1}(T) \lambda_{1}\left(\frac{1+\epsilon^{2}|\nabla v|^{2}}{(1+v)^{2}}\right)\left|\partial_{\eta} \phi_{v}(\cdot, \cdot, 1)\right|^{2}+\lambda_{2} P(T, v) .
$$

In the upcoming analysis we follow the approach of [LW16]. We can use similar arguments because the main difference between our operator for the electrostatics and the operator used in [LW16 lies in the lower order terms. Before we start our analysis by proving local well-posedness of the derived system equations 6.3 - 6.6), we have to make sure that the temperature dependent parameters satisfy suitable hypotheses needed for our analysis. However at first we need to introduce some notation:

Definition 6.1. Given $p \in[2, \infty]$, define

$$
W_{p, \mathcal{B}}^{\alpha}(E):=\left\{\begin{array}{c}
\left\{w \in W_{p}^{\alpha}(E): w=\partial_{r} w=0 \text { on } \delta E\right\}, \alpha \in(1+1 / p, \infty), \\
\left\{w \in W_{p}^{\alpha}(E): w=0 \text { on } \delta E\right\}, \alpha \in(1 / p, 1+1 / p), \\
W_{p}^{\alpha}(E), \alpha \in[0,1 / p)
\end{array}\right.
$$


and for $\kappa \in(0,1)$ define

$$
S_{p}(\kappa):=\left\{w \in W_{p, \mathcal{B}}^{2}(E):\|w\|_{W_{p, \mathcal{B}}^{2}}<1 / \kappa \text { and }-1+\kappa<w(x, y) \text { for }(x, y) \in E\right\}
$$

\subsection{Assumptions on the temperature dependence}

In the upcoming analysis we will always assume the following to be satisfied:

Definition 6.2. Let $X \subset \mathbb{R}^{2}$ and $Y \subset \mathbb{R}$ be open and bounded. We say that the assumption $\mathrm{C} 1(3 \mathrm{~d})$ is satisfied if the following holds:

$$
\| \begin{gathered}
\|\sigma(T(\cdot, \cdot, \cdot))\|_{L_{\infty}(\Omega)}<\infty, \\
\|\partial \sigma(\cdot)\|_{L_{\infty}(\mathbb{R})}<\infty, \\
\sigma(T(x, y, z)) \geq c>0, \quad(x, y, z) \in \Omega(u), \\
\epsilon_{1}: \mathbb{R} \rightarrow \mathbb{R}, \quad T \rightarrow \epsilon_{1}(T) \\
\text { is globally Lipschitz for } T \in Y, \\
P: \mathbb{R}^{2} \rightarrow \mathbb{R}, \quad(u, T) \rightarrow P(u, T) \\
\text { is globally Lipschitz for }(u, T) \in X
\end{gathered}
$$

We will get better results if we make an additional assumption on the physical parameters which govern the behaviour of the RHS $g$ in equation (6.6).

Definition 6.3. Let $\kappa \in(0,1)$ be given. We say the assumption C2 (3d) is satisfied with $b(\kappa)>0$, if $\epsilon_{1}, \lambda_{1}, \lambda_{2}$ and $P$ are such that there exists a $b(\kappa)$ with:

$$
\left\|\begin{array}{c}
\|g(v)\|_{L_{2}(E)} \leq b(\kappa), \text { for } v \in S_{3}(\kappa) \\
\|g(v)-g(w)\|_{L_{2}(E)} \leq b(\kappa)\|v-w\|_{W_{3}^{2}(E)}, \text { for } v, w \in S_{3}(\kappa)
\end{array}\right\|
$$

Remark 6.4. The interpretation of this assumption is that the thermal effects which push the membrane upward and the electrostatic effects which pull the membrane downward are equally strong and therefore cancel out each other. In models without temperature, global existence is usually proved under the assumption of a small voltage. In Theorem 6.13 it will become evident that assumption $\mathrm{C} 2(3 \mathrm{~d})$ is a possible replacement for that. For example $\mathrm{C} 2(3 \mathrm{~d})$ is satisfied if $P$ is given by the ideal gas law and both 
the amount of substance $N$ and the voltage $V$ are small. This is due to the Lipschitz continuity of $g$ which will be shown in Theorem 6.5 .

In order to solve the full coupled system we first solve the problem for the electrostatic potential under the assumption that the membrane's deflection $u$ is given.

\subsection{Well-posedness of the electrostatic problem}

We state the main result in this subsection:

Theorem 6.5. Suppose that assumption $C 1(3 d)$ is satisfied. Let $\kappa \in(0,1)$ and $q \geq 3$ be given and pick $v \in S_{q}(\kappa)$. For each $F \in L_{2}(\Omega)$, there is a unique solution $\phi_{v} \in W_{2}^{2}(\Omega)$ to the equations (6.3) and (6.4). Furthermore there is $c_{1}(\kappa)>0$ such that:

$$
\left\|\phi_{v_{1}}-\left.\phi_{v_{2}}\right|_{W_{2}^{2}(\Omega)} \leq c_{1}(\kappa)\right\| v_{1}-v_{2} \|_{W_{q}^{2}(E)}, \quad v_{1}, v_{2} \in S_{q}(\kappa)
$$

and the mapping

$$
g: S_{q}(\kappa) \rightarrow L_{2}(E), \quad v \rightarrow g(v)
$$

is globally Lipschitz continuous and bounded.

We want to prove Lemma 6.7 assuming only $T \in W_{2}^{2}$. We cannot assume that $T \in W_{\infty}^{1}$ holds because for $n=3$ the space $W_{2}^{2}$ is not embedded in $W_{\infty}^{1}$. This however leaves us with some unbounded coefficients in the operator $\mathfrak{L}_{(u, T)}$. Similar arguments, for example in ELW15,LW16, usually make use of the abstract well-posedness result GT01, Theorem 8.3]. Due to this situation we need a result with less restrictive assumptions:

Lemma 6.6. Denote by $\Omega^{\prime} \subset \mathbb{R}^{n}$ a bounded domain and by $L$ a strictly elliptic operator in divergence form:

$$
L u:=D_{i}\left(a^{i j(x)} D_{j} u+b^{i}(x) u\right)+c^{i}(x) D_{i} u+d(x) u, x \in \Omega^{\prime} .
$$

Let the operator L satisfy the following conditions:

$$
a^{i j} \in L_{\infty}\left(\Omega^{\prime}\right),
$$




$$
\left(b^{i}\right)^{2},\left(c^{i}\right)^{2}, d \in L_{q / 2}\left(\Omega^{\prime}\right),
$$

where $q>n$. Then for $\phi \in W_{2}^{1}\left(\Omega^{\prime}\right)$ and $g \in L_{2}\left(\Omega^{\prime}\right)$ the Dirichlet problem

$$
L u=g \text { in } \Omega^{\prime}, \quad u=\phi \text { on } \partial \Omega^{\prime}
$$

has a unique weak solution $u \in W_{2}^{1}\left(\Omega^{\prime}\right)$.

Proof. In order to apply the proof of GT01, Theorem 8.3] here, we need to replace GT01, Lemma 8.4] with the following estimate for unbounded $b^{i}, c^{i}, a$ which is related to the coercivity of $\mathcal{L}$, the to $L$ associated bilinear form:

$$
\mathcal{L}(u, u) \geq c_{1}\|\nabla u\|_{L_{2}}^{2}-c_{2}\|u\|_{L_{2}}^{2}
$$

This is done in [LU73, Chapter 3, Lemma 4.1] with the help of finer so-called multiplicative inequalities, that is inequalities of the type

$$
\|u\|_{L_{p}} \leq c\|\nabla u\|_{L_{q}}^{a}\|u\|_{L_{r}}^{b}, u \in W_{p}^{1}
$$

with $a, b, p, q, r, c>0$.

Finally Gri85, Theorem 3.2.1.2] will supply us with a strong solution for our convex domain. However for that we will have to formulate the equation governing the electrostatic potential with an operator that features Lipschitz coefficients and with a right-hand side in $L_{2}$. In order to prove Theorem 6.5 we need several preparatory Lemmas:

Lemma 6.7. Let $\kappa \in(0,1)$ and $q>2$ be given. For each $v \in \bar{S}_{q}(\kappa)$ and $F \in L_{2}(\Omega)$, there is a unique solution $\phi \in W_{2}^{1}(\Omega)$ to the boundary value problem:

$$
\begin{gathered}
\mathfrak{L}_{(v, T)} \phi=F, \quad \text { in } \Omega, \\
\phi=0, \quad \text { on } \partial \Omega .
\end{gathered}
$$




\section{Analysis of a $3 d$ model}

Proof. The definition of $\bar{S}_{q}(\kappa)$ and Sobolev's embedding theorem guarantee the existence of some constant $c_{0}>0$ such that for $v \in \bar{S}_{q}(\kappa)$,

$$
1+v(x, y) \geq \kappa,(x, y) \in E,\|v\|_{C^{1}(\bar{E})} \leq \frac{c_{0}}{\kappa}
$$

First, we write our operator in divergence form,

$$
\begin{aligned}
\mathfrak{L}_{(v, T)} w= & \partial_{x}\left(a_{11}(v, T) \partial_{x} w+a_{13}(v, T) \partial_{\eta} w\right)+\partial_{y}\left(a_{22}(v, T) \partial_{y} w+a_{23}(v, T) \partial_{\eta} w\right) \\
& +\partial_{\eta}\left(a_{31}(v, T) \partial_{x} w+a_{32}(v, T) \partial_{y} w+a_{33}(v, T) \partial_{\eta} w\right) \\
& +b_{1}(v, T) \partial_{x} w+b_{2}(v, T) \partial_{y} w+b_{3}(v, T) \partial_{\eta} w,
\end{aligned}
$$

with

$$
\begin{aligned}
& a_{11}(v, T):=\epsilon^{2} \sigma(T), \quad a_{13}(v, T):=a_{31}(v, T):=-\epsilon^{2} \eta \frac{\partial_{x} v}{1+v} \sigma(T), \\
& a_{22}(v, T):=\epsilon^{2} \sigma(T), \quad a_{23}(v, T):=a_{32}(v, T):=-\epsilon^{2} \eta \frac{\partial_{y} v}{1+v} \sigma(T), \\
& a_{33}(v, T):=\frac{1+\epsilon^{2} \eta^{2}|\nabla v|^{2}}{(1+v)^{2}} \sigma(T), \\
& b_{1}(v, T):=\epsilon^{2} \partial \sigma(T)\left(\partial_{x} T-\partial_{\eta} T \frac{\eta \partial_{x} v}{1+v}\right)+\epsilon^{2} \frac{\partial_{x} v}{1+v}, \\
& b_{2}(v, T):=\epsilon^{2} \partial \sigma(T)\left(\partial_{y} T-\partial_{\eta} T \frac{\eta \partial_{y} v}{1+v}\right)+\epsilon^{2} \frac{\partial_{y} v}{1+v} \\
& b_{3}(v, T):=-\epsilon^{2} \eta \frac{|\nabla v|^{2}}{(1+v)^{2}}
\end{aligned}
$$

The principle part of the operator is given by

$$
\begin{aligned}
\mathfrak{L}_{(v, T)}^{0} w= & \partial_{x}\left(a_{11}(v, T) \partial_{x} w+a_{13}(v, T) \partial_{\eta} w\right)+\partial_{y}\left(a_{22}(v, T) \partial_{y} w+a_{23}(v, T) \partial_{\eta} w\right) \\
& +\partial_{\eta}\left(a_{31}(v, T) \partial_{x} w+a_{32}(v, T) \partial_{y} w+a_{33}(v, T) \partial_{\eta} w\right) .
\end{aligned}
$$

Since we assumed that $\sigma(T) \geq c>0$ in C1 (3d) we can argue just like in [LW16, Lemma 2.2] that the operator $\mathfrak{L}_{(u, T)}$ is elliptic with ellipticity constant $\mu(\kappa)>0.5$ Because of assumption $\mathrm{C} 1(3 \mathrm{~d}), 6.12$ and $T \in W_{2}^{2}(\Omega)$ the claim now follows from Lemma 6.6 since

\footnotetext{
${ }^{5}$ The principle part of our operator and the one in LW16 only differ by the factor $\sigma(T)$.
} 
the Sobolev embedding

$$
W_{2}^{1}(\Omega) \hookrightarrow L_{6}(\Omega)
$$

holds true for $n=3$.

The following results show that if we start with smoother functions for the deflection we will get smoother solutions for the electrostatic potential as well.

Lemma 6.8. Let $\kappa \in(0,1)$ be given. For each $v \in \bar{S}_{\infty}(\kappa)$ and $F \in L_{2}(\Omega)$, the unique solution $\phi \in W_{2}^{1}(\Omega)$ to the boundary value problem in Lemma 6.7 belongs to $W_{2}^{2}(\Omega)$.

Proof. The regularity of $v, T$ and $\phi$ ensures that:

$$
G:=F+b_{1}(v, T) \partial_{x} \phi+b_{2}(v, T) \partial_{y} \phi+b_{3}(v, T) \partial_{\eta} \phi \in L_{2}(\Omega)
$$

Furthermore $v \in \bar{S}_{\infty}(\kappa)$ implies that $a_{i j}(v, T) \in W_{\infty}^{1}$ for $1 \leq i, j \leq 3$. Therefore the conditions in Gri85, 3.1.3] are satisfied and we can apply Gri85, Theorem 3.2.1.2] which yields that the boundary value problem

$$
\mathfrak{L}_{(v, T)}^{0} \widehat{\phi}=G \text { in } \Omega, \quad \widehat{\phi}=0 \text { on } \partial \Omega
$$

has a unique solution $\widehat{\phi} \in W_{2}^{2}(\Omega)$. Applying GT01, Theorem 8.3 to 6.13 shows that 6.13 has a weak solution in $W_{2}^{2}(\Omega)$. Due to the definition of $G, \phi$ and $\widehat{\phi}$ and Lemma 6.7 we have $\phi=\widehat{\phi} \in W_{2}^{2}(\Omega)$.

The next two Lemmas proofs can be found in LW16] and will help us to prove Lemma 6.11

Lemma 6.9. Let $p \in[2,4]$ be given. There exists $c_{3}(p)>0$ such that

$$
\|w(\cdot, \cdot, 1)\|_{L_{p}(E)}^{p} \leq c_{3}(p)\|w\|_{W_{2}^{1}(\Omega)}^{(3 p-4) / 2}\|w\|_{L_{2}(\Omega)}^{(4-p) / 2}, \quad w \in W_{2}^{1}(\Omega)
$$

Lemma 6.10. If $\phi \in W_{2}^{2}(\Omega)$ and $\phi=0$ on $\partial \Omega$, then

$$
\int_{\Omega} \partial_{x}^{2} \phi \partial_{\eta}^{2} \phi d(x, y, \eta)=\int_{\Omega}\left|\partial_{x} \partial_{\eta} \phi\right|^{2} d(x, y, \eta)
$$


and

$$
\int_{\Omega} \partial_{y}^{2} \phi \partial_{\eta}^{2} \phi d(x, y, \eta)=\int_{\Omega}\left|\partial_{y} \partial_{\eta} \phi\right|^{2} d(x, y, \eta) .
$$

In the following we first derive an estimate for $\|\phi\|_{W_{2}^{1}(\Omega)}$. After that we can estimate $\|\phi\|_{W_{2}^{2}(\Omega)}$. The next Lemma will allow us to prove $L_{2}$ regularity for some of the 2nd order terms in our operator $\mathfrak{L}_{(v, T)}$.

Lemma 6.11. Let $\kappa \in(0,1)$ and $q>2$ be given. For each $v \in \bar{S}_{q}(\kappa)$ and $F \in L_{2}(\Omega)$, the weak solution $\phi \in W_{2}^{1}(\Omega)$ to the boundary value problem 6.10, 6.11 belongs to the space $X(\Omega)$ defined by

$$
X(\Omega):=\left\{w \in W_{2}^{1}(\Omega): \partial_{\eta} w \in W_{2}^{1}(\Omega)\right\}
$$

Furthermore there is $c_{4}(\kappa)>0$ such that

$$
\|\phi\|_{W_{2}^{1}(\Omega)}+\left\|\partial_{\eta} \phi\right\|_{W_{2}^{1}(\Omega)} \leq c_{4}(\kappa)\|F\|_{L_{2}(\Omega)}
$$

Proof. First we define

$$
P_{x}:=\partial_{x} \phi-\eta \frac{\partial_{x} v}{1+v} \partial_{\eta} \phi, \quad P_{y}:=\partial_{y} \phi-\eta \frac{\partial_{y} v}{1+v} \partial_{\eta} \phi, \quad P_{\eta}:=\frac{\partial_{\eta} \phi}{1+v}
$$

Invoking 6.12 and using assumption C1 (3d) we find

$$
\|\nabla \phi\|_{L_{2}(\Omega)}^{2} \leq c(\kappa)\left[\epsilon^{2}\left\|P_{x}\right\|_{L_{2}(\Omega)}^{2}+\epsilon^{2}\left\|P_{y}\right\|_{L_{2}(\Omega)}^{2}+\left\|P_{\eta}\right\|_{L_{2}(\Omega)}^{2}\right]
$$

Furthermore applying assumption C1 (3d), Hölder's inequality, $W_{2}^{1}(\Omega) \hookrightarrow L_{6}(\Omega)$, Young's inequality and lastly [LU73, Chapt. 3.4 (4.19)] yields

$$
\begin{aligned}
& \int_{\Omega} \frac{\phi \mathfrak{L}_{(u, T)}^{2} \phi}{\sigma(T)} d(x, y, \eta) \\
& \leq c\|\phi\|_{L_{3}}\left\|\partial_{x} T\right\|_{L_{6}}\|\nabla \phi\|_{L_{2}} \\
& \leq c\left[\frac{\epsilon_{1}}{2}\|\phi\|_{L_{3}}^{2}+\frac{1}{2 \epsilon_{1}}\|\nabla \phi\|_{L_{2}}^{2}\right] \\
& \leq c^{\prime}\left[\frac{\epsilon_{1} \epsilon_{2}}{2}\|\nabla \phi\|_{L_{2}}^{2}+\frac{\epsilon_{1} c\left(\epsilon_{2}\right)}{2}\|\phi\|_{L_{2}}^{2}+\frac{1}{2 \epsilon_{1}}\|\nabla \phi\|_{L_{2}}^{2}\right]
\end{aligned}
$$




\section{Analysis of a $3 d$ model}

with constants $c, c^{\prime}, c\left(\epsilon_{2}\right)>0$. Choosing $\epsilon_{1}>0$ big enough and then $\epsilon_{2}>0$ small enough we find that 6.17) implies:

$$
\int_{\Omega} \frac{\phi \mathfrak{L}_{(u, T)}^{2} \phi}{\sigma(T)} d(x, y, \eta) \leq c^{\prime}(\kappa)\left(\|\phi\|_{L_{2}(\Omega)}^{2}+\frac{\epsilon^{2}}{4}\left(\left\|P_{x}\right\|_{L_{2}(\Omega)}^{2}+\left\|P_{y}\right\|_{L_{2}(\Omega)}^{2}+\left\|P_{\eta}\right\|_{L_{2}(\Omega)}^{2}\right) .\right.
$$

By using the splitting $\mathfrak{L}_{(u, T)}=\sigma(T) \mathfrak{L}_{(u, T)}^{1}+\mathfrak{L}_{(u, T)}^{2}$ and then invoking 6.10 and the Divergence Theorem for the term with $\mathfrak{L}_{(u, T)}^{1}$ we find:

$$
\begin{aligned}
& \epsilon^{2}\left\|P_{x}\right\|_{L_{2}(\Omega)}^{2}+\epsilon^{2}\left\|P_{y}\right\|_{L_{2}(\Omega)}^{2}+\left\|P_{\eta}\right\|_{L_{2}(\Omega)}^{2} \\
& =\int_{\Omega} \frac{F \phi}{\sigma(T)} d(x, y, \eta)+\epsilon^{2} \int_{\Omega}\left[\frac{\partial_{x} v}{1+v} \phi P_{x}+\frac{\partial_{y} v}{1+v} \phi P_{y}\right] d(x, y, \eta)+\int_{\Omega} \frac{\phi \mathfrak{L}_{(u, T)}^{2} \phi}{\sigma(T)} d(x, y, \eta) .
\end{aligned}
$$

We can now estimate the latter quantity:

$$
\begin{aligned}
& \int_{\Omega} \frac{F \phi}{\sigma(T)} d(x, y, \eta)+\epsilon^{2} \int_{\Omega}\left[\frac{\partial_{x} v}{1+v} \phi P_{x}+\frac{\partial_{y} v}{1+v} \phi P_{y}\right] d(x, y, \eta)+\int_{\Omega} \frac{\phi \mathfrak{L}_{(u, T)}^{2} \phi}{\sigma(T)} d(x, y, \eta) \\
& \leq \frac{\epsilon^{2}}{2}\left[\left\|P_{x}\right\|_{L_{2}(\Omega)}^{2}+\left\|P_{y}\right\|_{L_{2}(\Omega)}^{2}+\left\|P_{\eta}\right\|_{L_{2}(\Omega)}^{2}\right]+c^{\prime \prime}(\kappa)\left[\|F\|_{L_{2}(\Omega)}^{2}+\|\phi\|_{L_{2}(\Omega)}^{2}\right] .
\end{aligned}
$$

To see that this holds true, we first observe that due to assumption C1 (3d) the estimate for the first two terms is already done in [LW16, proof Lemma 2.5] by Cauchy-Schwarz' and Young's inequality. For the leftover term, which is due to our operator being different from the one in [LW16], we can simply use 6.18). Now 6.17) yields

$$
\|\phi\|_{W_{2}^{1}(\Omega)}^{2} \leq c^{\prime \prime \prime}(\kappa)\left[\|F\|_{L_{2}(\Omega)}^{2}+\|\phi\|_{L_{2}(\Omega)}^{2}\right]
$$

which can be improved to

$$
\|\phi\|_{W_{2}^{1}(\Omega)}^{2} \leq c^{\prime \prime \prime \prime}(\kappa)\|F\|_{L_{2}(\Omega)}^{2} .
$$

The latter is due to a simple argument by contradiction. Details can be found in the proof of ELW14, Proposition 6, Eq(19)]. Now we turn to the estimate for $\partial_{\eta} \phi$ : We 
assume that $v$ also belongs to $\bar{S}_{\infty}\left(\kappa^{\prime}\right)$ for some $\kappa^{\prime} \in(0,1)$. We define

$$
\mathfrak{c}_{x}:=\partial_{x} \partial_{\eta} \phi, \quad \mathfrak{c}_{y}:=\partial_{y} \partial_{\eta} \phi, \quad \mathfrak{c}_{\eta}:=\partial_{\eta}^{2} \phi
$$

It follows from Lemma 6.10 that

$$
\int_{\Omega} \partial_{x}^{2} \phi \partial_{\eta}^{2} \phi d(x, y, \eta)=\int_{\Omega}\left|\mathfrak{c}_{x}\right|^{2} d(x, y, \eta), \quad \int_{\Omega} \partial_{y}^{2} \phi \partial_{\eta}^{2} \phi d(x, y, \eta)=\int_{\Omega}\left|\mathfrak{c}_{y}\right|^{2} d(x, y, \eta)
$$

With the definitions

$$
Q_{x}:=\mathfrak{c}_{x}-\eta \frac{\partial_{x} v}{1+v} \mathfrak{c}_{\eta}, \quad Q_{y}:=\mathfrak{c}_{y}-\eta \frac{\partial_{y} v}{1+v} \mathfrak{c}_{\eta}, \quad Q_{\eta}:=\frac{1}{1+v} \mathfrak{c}_{\eta}
$$

the estimate 6.12 and assumption $\mathrm{C1}(3 \mathrm{~d})$ imply

$$
\left\|\nabla \partial_{\eta} \phi\right\|_{L_{2}(\Omega)}^{2} \leq c(\kappa)\left[\left\|Q_{x}\right\|_{L_{2}(\Omega)}^{2}+\left\|Q_{y}\right\|_{L_{2}(\Omega)}^{2}+\left\|Q_{\eta}\right\|_{L_{2}(\Omega)}^{2}\right]
$$

and

$$
\int_{\Omega} \frac{\partial_{\eta}^{2} \phi \mathfrak{L}_{(u, T)}^{2} \phi}{\sigma(T)} d(x, y, \eta) \leq c^{\prime}(\kappa)\left\|\partial_{\eta} \phi\right\|_{L_{2}(\Omega)}^{2}+\frac{\epsilon^{2}}{4}\left(\left\|Q_{x}\right\|_{L_{2}(\Omega)}^{2}+\left\|Q_{y}\right\|_{L_{2}(\Omega)}^{2}+\epsilon^{-2}\left\|Q_{\eta}\right\|_{L_{2}(\Omega)}^{2}\right) .
$$

To see this, one uses (6.12 and assumption C1 (3d) to get rid of the terms which include $v$ and $T$. Then one applies Cauchy Schwarz' and Young's inequality. Now we can estimate:

$$
\begin{aligned}
& \epsilon^{2}\left\|Q_{x}\right\|_{L_{2}(\Omega)}^{2}+\epsilon^{2}\left\|Q_{y}\right\|_{L_{2}(\Omega)}^{2}+\left\|Q_{\eta}\right\|_{L_{2}(\Omega)}^{2} \\
& =\int_{\Omega} \frac{F \mathfrak{c}_{\eta}}{\sigma(T)} d(x, y, \eta)-\frac{\epsilon^{2}}{2} \int_{\Omega}\left(\frac{2|\nabla v|^{2}-(1+v) \Delta v}{(1+v)^{2}}\right)\left|\partial_{\eta} \phi\right|^{2} d(x, y, \eta) \\
& +\frac{\epsilon^{2}}{2} \int_{E}\left(\frac{2|\nabla v|^{2}-(1+v) \Delta v}{(1+v)^{2}}\right)\left|\partial_{\eta} \phi(x, y, 1)\right|^{2} d(x, y)+\int_{\Omega} \frac{\partial_{\eta}^{2} \phi \mathfrak{L}_{(u, T)}^{2} \phi}{\sigma(T)} d(x, y, \eta) \\
& \leq \frac{\epsilon^{2}}{2}\left[\left\|Q_{x}\right\|_{L_{2}(\Omega)}^{2}+\left\|Q_{y}\right\|_{L_{2}(\Omega)}^{2}+\epsilon^{-2}\left\|Q_{\eta}\right\|_{L_{2}(\Omega)}^{2}\right]+c^{\prime \prime}(\kappa)\left[\|F\|_{L_{2}(\Omega)}^{2}+\left\|\partial_{\eta} \phi\right\|_{L_{2}(\Omega)}^{2}\right] .
\end{aligned}
$$

The identity in the first line is again due to the splitting $\mathfrak{L}_{(u, T)}=\sigma(T) \mathfrak{L}_{(u, T)}^{1}+\mathfrak{L}_{(u, T)}^{2}$, 6.10, 6.21 and $\partial_{\eta} \phi \partial_{\eta}^{2} \phi=\partial_{\eta}\left(\left|\partial_{\eta} \phi\right|^{2}\right) / 2$. For the estimate in the last line we first observe 


\section{Analysis of a $3 d$ model}

that the estimate for the first three terms is already done in [LW16, proof Lemma 2.5] and can be used without change because C1 (3d) is satisfied. For the leftover term we can simply use 6.23). Combining this with 6.22 gives

$$
\left\|\partial_{\eta} \phi\right\|_{W_{2}^{1}(\Omega)}^{2} \leq c^{\prime \prime \prime}(\kappa)\left[\|F\|_{L_{2}(\Omega)}^{2}+\left\|\partial_{\eta} \phi\right\|_{L_{2}(\Omega)}^{2}\right]
$$

Since our estimate is independent of the regularity of $v$ the claim for all $v \in \bar{S}_{q}(\kappa)$ follows by approximation.

Now we want to prove the $W_{2}^{2}$ regularity of our solution under the assumption of slightly smoother functions $v$ (see [LW16, Proposition 2.7]):

Lemma 6.12. Let $\kappa \in(0,1)$ and $q \geq 3$ be given. For each $v \in \bar{S}_{q}(\kappa)$ and $F \in L_{2}(\Omega)$, the weak solution $\phi \in W_{2}^{1}(\Omega)$ to the boundary value problem (6.10), (6.11) belongs to $W_{2}^{2}(\Omega)$ and there is $c_{5}(\kappa)>0$ such that

$$
\|\phi\|_{W_{2}^{2}(\Omega)} \leq c_{5}(\kappa)\|F\|_{L_{2}(\Omega)}
$$

Proof. First we define

$$
\begin{aligned}
J_{1}:= & 2 \epsilon^{2} \eta \frac{\partial_{x} u}{1+u} \partial_{x} \partial_{\eta} \phi+2 \epsilon^{2} \eta \frac{\partial_{y} u}{1+u} \partial_{y} \partial_{\eta} \phi \\
& +\left(1-\frac{1+\epsilon^{2} \eta^{2}|\nabla u|^{2}}{(1+u)^{2}}\right) \partial_{\eta}^{2} \phi-\epsilon^{2} \eta\left[2 \frac{|\nabla u|^{2}}{(1+u)^{2}}-\frac{\Delta u}{1+u}\right] \partial_{\eta} \phi
\end{aligned}
$$

and

$$
\begin{aligned}
J_{2}:= & -\left(\epsilon ^ { 2 } \eta \partial \sigma ( T ) \left[-\frac{\partial_{x} u}{1+u}\left(\partial_{x} T-\frac{\eta \partial_{x} u}{1+u} \partial_{\eta} T\right)\right.\right. \\
& \left.\left.-\frac{\partial_{y} u}{1+u}\left(\partial_{y} T-\frac{\eta \partial_{y} u}{1+u} \partial_{\eta} T\right)\right]+\frac{\partial \sigma(T) \partial_{\eta} T}{(1+u)^{2}}\right) \partial_{\eta} \phi \\
& -\epsilon^{2} \partial \sigma(T)\left(\partial_{x} T-\partial_{\eta} T \frac{\eta \partial_{x} u}{1+u}\right) \partial_{x} \phi-\epsilon^{2} \partial \sigma(T)\left(\partial_{y} T-\partial_{\eta} T \frac{\eta \partial_{y} u}{1+u}\right) \partial_{y} \phi
\end{aligned}
$$

We introduce the following convenient splitting:

$$
J:=J_{1}+J_{2} \sigma(T)^{-1} .
$$




\section{Analysis of a $3 d$ model}

Again $J_{1}$ denotes the same term as in $\overline{\mathrm{LW} 16}$ and $J_{2}$ is what we get in addition to that due to our different operator. Thus we can simply reorder the boundary value problem for $\phi$ to get

$$
\epsilon^{2} \partial_{x} \phi+\epsilon^{2} \partial_{y} \phi+\partial_{\eta} \phi=J, \quad \phi=0 \text { on } \partial \Omega
$$

Now we apply Lemma 6.11 and the continuous embeddings of $W_{q}^{2}(E)$ in $W_{\infty}^{1}(E)$ and $W_{2}^{1}(\Omega)$ in $L_{6}(\Omega)$ (for the terms with second derivatives of $v$ ) to obtain

$$
\left\|\left.J\right|_{L_{2}(\Omega)} \leq c(\kappa)\right\| F \|_{L_{2}(\Omega)} .
$$

For the terms which include $T$ we used assumption $\mathrm{C} 1(3 \mathrm{~d})$ and $T \in W_{3}^{2}(\Omega)$. Now we infer from Gri85, Theorem 3.2.1.2] that $\phi \in W_{2}^{2}(\Omega)$. It remains to prove that

$$
\|\phi\|_{W_{2}^{2}(\Omega)} \leq c(\kappa)\|J\|_{L_{2}(\Omega)}
$$

holds true. Due to our preparation this last step can be found in LW16, proof Proposition 2.7]: After invoking Gri85, Theorem 3.1.3.1 \& Lemma 3.2.1.1] we can use the approximation in Gri85, Theorem 3.2.1.2].

Now we are ready to proof the well-posedness result:

Proof of Theorem 6.5. We set

$$
\begin{aligned}
h & :=-\mathfrak{L}_{(v, T)} \eta=\epsilon^{2} \eta\left[2 \frac{|\nabla u|^{2}}{(1+u)^{2}}-\frac{\Delta u}{1+u}\right] \\
& +\left(\epsilon^{2} \eta \partial \sigma(T)\left[-\frac{\partial_{x} u}{1+u}\left(\partial_{x} T-\frac{\eta \partial_{x} u}{1+u} \partial_{\eta} T\right)-\frac{\partial_{y} u}{1+u}\left(\partial_{y} T-\frac{\eta \partial_{y} u}{1+u} \partial_{\eta} T\right)\right]+\frac{\partial \sigma(T) \partial_{\eta} T}{(1+u)^{2}}\right) .
\end{aligned}
$$

Since $v \in S_{q}(\kappa)$ and $\mathrm{C} 1(3 \mathrm{~d})$ is satisfied, $h$ belongs to $L_{2}(\Omega)$ and therefore Lemma 6.12 ensures that there is a unique solution $\phi_{v}$ to

$$
\mathfrak{L}_{(v, T)} \phi_{v}=h \text { in } \Omega, \quad \phi_{v}=0 \text { on } \partial \Omega
$$

satisfying

$$
\left\|\phi_{v}\right\|_{W_{2}^{2}(\Omega)} \leq c_{5}(\kappa)\|f\|_{L_{2}(\Omega)}
$$


Setting $\psi_{v}:=\phi_{v}-\eta$, the function $\psi_{v}$ obviously solves 6.4 and satisfies

$$
\left\|\psi_{v}\right\|_{W_{2}^{2}(\Omega)} \leq c_{7}(\kappa)
$$

Lastly, by assumption $\mathrm{C} 1(3 \mathrm{~d})$ the proof of the equations 6.8 and $(6.9)$ is identical to the proof in [LW16, Proposition 2.1].

\subsection{Well-posedness of the deflection problem}

Having proved the well-posedness of the electrostatic potential for a given deflection, we can now prove well-posedness of the system by solving the underlying non-linear equation for the evolution of the membrane.

\section{Theorem 6.13.}

Suppose that $C 1$ (3d) is satisfied. Let $\epsilon>0, \mathcal{T} \in B U C^{1}(\Omega)$ and $4 \xi \in(7 / 3,4)$ be given. For the membrane's displacement consider an initial value $u_{0} \in W_{2}^{4 \xi}(E)$ such that $u_{0}=\partial_{r} u_{0}=0$ on $\partial E$ and $u_{0}(x)>-1$ for $x \in E$. Then the following holds true:

1. There is a unique solution $(u, \psi)$ to the full system on the maximal interval of existence $\left[0, T_{m}\right)$ in the sense that

$$
u \in C^{1}\left(\left(0, T_{m}\right), L_{2}(E)\right) \cap C\left(\left[0, T_{m}\right), W_{2}^{4 \xi}(E)\right)
$$

satisfies equations (6.5) and (6.6), while $\psi(t) \in W_{2}^{2}(\Omega(u(t))$ also solves the corresponding equation in $\Omega(u(t))$ for each $t \in\left[0, T_{m}\right)$.

2. Given $\kappa \in(0,1)$ there is $r(\kappa)>0$ and $b(\kappa)>0$ such that $T_{m}=\infty$, provided that $\left\|u_{0}\right\|_{W_{q}^{2}(E)} \leq r(\kappa)$ and that $C 2(3 d)$ is satisfied with $b(\kappa)>0$.

Proof. Observe that $W_{2}^{4 \xi}$ is continuously embedded in $W_{3}^{2}$. This implies that

$$
\|w\|_{W_{3}^{2}(E)} \leq \bar{c}\|w\|_{W_{2}^{4 \xi}(E)}, \quad w \in W_{3}^{2}(E)
$$

for $\bar{c}>1$. Furthermore since $u_{0} \in W_{2}^{4 \xi}$ we can chose $\kappa \in(0,1)$ such that $u_{0} \in S_{3}(2 \kappa)$ 


\section{Analysis of a $3 d$ model}

and $\left\|u_{0}\right\|_{W_{2}^{4 \xi}(E)} \leq 1 /(2 \kappa)$. Now let

$$
A w:=\lambda_{6} D \nabla_{\perp}^{4} w-\mu \nabla_{\perp}^{2} w, \quad w \in W_{2}^{4}
$$

and recall that $-A$ generates an exponentially decaying analytic semigroup on $L_{2}(E)$ with

$$
\left\|e^{-t A}\right\|_{\mathcal{L}\left(W_{2, D}^{4 \xi}(E)\right)}+\left\|e^{-t A}\right\|_{\mathcal{L}\left(W_{2, D}^{1}(E)\right)}+t^{\xi}\left\|e^{-t A}\right\|_{\mathcal{L}\left(L_{2}(E), W_{2, D}^{4 \xi}(E)\right)} \leq M e^{-\nu t}
$$

where $\nu>0, M \geq 1, t>0$.

We now invoke Theorem 6.5 to find that $g$ is well defined on $S_{3}(\kappa)$ and that there is $C_{1}(\kappa)>0$ such that

$$
\|g(v)\|_{L_{2}(E)} \leq C_{1}(\kappa)\|v\|_{W_{3}^{2}(E)}, \quad v \in \bar{S}_{3}(\kappa)
$$

and

$$
\left\|g\left(v_{1}\right)-g\left(v_{2}\right)\right\|_{L_{2}(E)} \leq C_{1}(\kappa)|| v_{1}-v_{2} \|_{W_{3}^{2}(E)}, \quad v_{1}, v_{2} \in \bar{S}_{3}(\kappa)
$$

holds true. Therefore we can rewrite the problem for the deflection of the membrane as the semilinear Cauchy problem

$$
\partial_{t} u+A u=g(u), t>0, u(0)=u_{0} .
$$

For $\tau>0$ we define the space

$$
\mathcal{V}_{\tau}(\kappa):=\left\{v \in C\left([0, \tau], \bar{S}_{3}(\kappa)\right)\right\}
$$

We endow $\mathcal{V}_{\tau}(\kappa)$ with the metric induced by $C\left([0, \tau], \bar{S}_{3}\left(\kappa_{0}\right)\right)$ where $\kappa_{0}:=\kappa / M \bar{c}$. The following approach is also used in [ELW14], [ELW15] and [LW16]: We show that

$$
\Gamma(v)(t):=e^{-t A} u_{0}+\int_{0}^{t} e^{-(t-s)} g(v(s)) d s, t \in[0, \tau], v \in V_{\tau}\left(\kappa_{0}\right)
$$

defines a contraction from $\mathcal{V}_{\tau}(\kappa)$ into itself. We now assume that $\tau$ is sufficiently small 
and consider $v_{1}, v_{2} \in \mathcal{V}_{\tau}(\kappa)$ and $t \in[0, \tau]$. Using $u_{0} \in S_{3}(2 \kappa), 6.29$ and 6.28 yields

$$
\Gamma\left(v_{1}\right)(t) \geq-1+2 \kappa
$$

Invoking 6.28) and 6.29) implies

$$
\left\|\Gamma\left(v_{1}\right)(t)\right\|_{W_{3}^{2}} \leq \frac{1}{2 \kappa}
$$

Lastly, by applying (6.28) and 6.30 we find

$$
\left\|\Gamma\left(v_{1}\right)(t)-\Gamma\left(v_{2}\right)(t)\right\|_{W_{3}^{2}} \leq c(\kappa, \tau)\left\|v_{1}-v_{2}\right\|_{\mathcal{V}_{\tau}(\kappa)}
$$

with $0<c(\kappa, \tau)<1$. Consequently $\Gamma$ defines a contraction from $\mathcal{V}_{\tau}(\kappa)$ into itself. Therefore $\Gamma(v)$ has a unique fixed point which is a solution to 6.31. For part 2 of Theorem 6.13 we simply use assumption $\mathrm{C} 2(3 \mathrm{~d})$ instead of $\tau$ being sufficiently small.

Remark 6.14. One also ends up with global existence if instead of C2 (3d) one assumes that $z:=\frac{a^{2}}{h}$ is sufficiently small. In that case $z$ assumes the role of the parameter $\lambda$ in [ELW15]. This condition can be interpreted as the cylinder being sufficiently thin. 


\section{Analysis of a $2 d$ model}

In this section we propose and analyse an alternative simplification of the full model.

\subsection{The model}

In this model we do not assume that the temperature $\mathcal{T}_{u}$ is given and instead let it be modelled by the respective PDE. Also we let the shear modulus $\mu\left(\mathcal{T}_{u}\right)$ still depend on temperature.

However we do make the following simplifications: We assume that tension dominates

rigidity, that means $\frac{D}{\mu} \ll 1$. Thus we can omit the 4 th spatial derivative in the equation for the membrane's displacement. This is a standard simplification which is used for example in PB02, ELW14, GPW05, EGG10]. This assumption is valid for micropumps and other MEMS devices that are described in the mentioned references. Also we assume that there is no variation in the horizontal direction orthogonal to the $x$-direction. Furthermore we discard the time-evolution for the temperature equation, that means we consider a regime where damping or thermal conductivity is high. Also we assume that the electric conductivity $\sigma\left(\mathcal{T}_{u}\right)=\sigma$ is independent of $\mathcal{T}_{u}$, which is for example a widely used assumption for gases. Lastly, we impose Dirichlet boundary conditions for the temperature. We fix an ambient temperature $\mathcal{T}_{0} \in C^{\infty}\left(\mathbb{R}^{2}, \mathbb{R}\right)$ and impose:

$$
\mathcal{T}_{u}(x, z)=\mathcal{T}_{0}(x, z), \quad(x, z) \in \partial \Omega(u(t))
$$

Just like in the $3 \mathrm{~d}$ case of section 6 we transform the problem to a fixed domain. Since we are in the $2 \mathrm{~d}$ case we make the following changes to the definitions of the ground plate and region:

$$
\begin{gathered}
E:=(-1,1) \\
\Omega(u(t)):=\{(x, z) \in E \times(-1, \infty):-1<z<u(x, t)\} .
\end{gathered}
$$

The volume is given by

$$
V_{C}=2+\int_{1}^{-1} u d x
$$




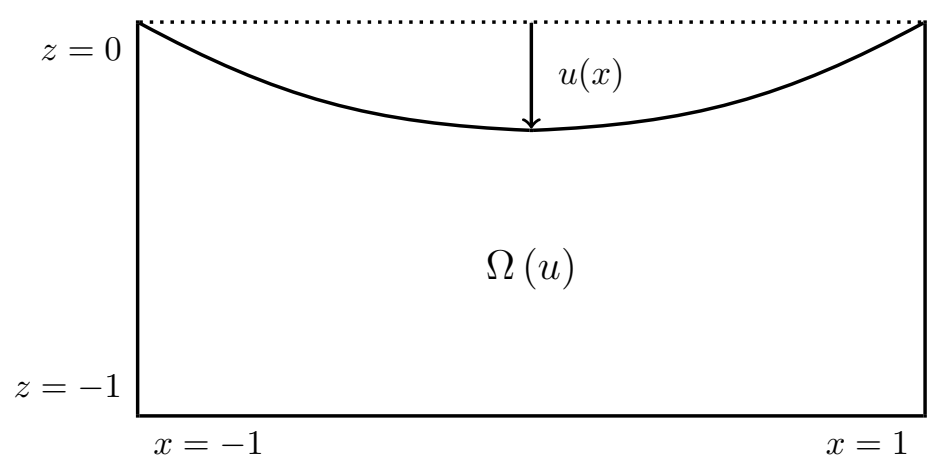

Figure 4: Domain $\Omega(u)$ in 2d.

The transformation map to the fixed domain $\Omega:=E \times(-1,0)$ is given by

$$
D_{u}(x, z):=\left(x, \frac{1+z}{1+u(x)}\right), \quad(x, z) \in \overline{\Omega(u)}
$$

with the inverse

$$
D_{u}^{-1}(x, \eta):=(x,(1+u(x)) \eta-1), \quad(x, \eta) \in \bar{\Omega} .
$$

Just as in the $3 \mathrm{~d}$ case we now restate the problem with

$$
\phi_{u}=\psi_{u} \circ D_{u}^{-1}, \quad T_{u}=\mathcal{T}_{u} \circ D_{u}^{-1}
$$

The deflection of the membrane $u$ is governed by

$$
\begin{gathered}
\partial_{t} u-\mu\left(T_{u}(\cdot, 1)\right) \partial_{x}^{2} u=-g\left(u, T_{u}(\cdot, 1), \phi_{u}(\cdot, 1)\right), \quad x \in E \\
u(x, t)=1, \text { for } x \in \partial E, t \in(0, \infty) \text { and } u(x, 0)=u_{0} \text { for } x \in E,
\end{gathered}
$$

with

$$
g\left(u, T_{u}, \phi_{u}\right):=\epsilon_{1}\left(\partial_{\eta} T_{u}, T_{u}\right) \lambda_{1} \frac{1+\epsilon^{2}\left|\partial_{x} u\right|^{2}}{(1+u)^{2}}\left|\partial_{\eta} \phi_{u}\right|^{2}-\lambda_{2} P\left(\partial_{\eta} T_{u}, T_{u}, u\right)
$$

The problem for the electrostatic potential $\phi_{u}$ is:

$$
\mathfrak{L}_{u} \phi_{u}(x, \eta)=0, \quad(x, \eta) \in \Omega,
$$




$$
\phi_{u}(x, \eta)=\eta, \quad(x, \eta) \in \partial \Omega
$$

with

$$
\begin{aligned}
& \mathfrak{L}_{u} w:=\epsilon^{2} \partial_{x}^{2} w-2 \epsilon^{2} \eta \frac{\partial_{x} u}{1+u} \partial_{x} \partial_{\eta} w \\
& +\frac{1+\epsilon^{2} \eta^{2}\left|\partial_{x} u\right|^{2}}{(1+u)^{2}} \partial_{\eta}^{2} w+\epsilon^{2} \eta\left[2 \frac{\left|\partial_{x} u\right|^{2}}{(1+u)^{2}}-\frac{\partial_{x}^{2} u}{1+u}\right] \partial_{\eta} w .
\end{aligned}
$$

Finally the temperature $T_{u}$ is governed by

$$
\begin{gathered}
\mathfrak{L}_{u} T_{u}=-h_{\left(u, \phi_{u}\right)}, \\
T_{u}(x, \eta)=\mathcal{T}_{0} \circ D_{u}^{-1}(x, \eta)=: T_{u, 0}(x, \eta), \quad(x, \eta) \in \partial \Omega,
\end{gathered}
$$

with

$$
h_{\left(u, \phi_{u}\right)}:=\sigma \lambda_{4}\left(\epsilon^{2}\left|\partial_{x} \phi_{u}\right|^{2}+\frac{1+\epsilon^{2} \eta^{2}\left|\partial_{x} u\right|^{2}}{(1+u)^{2}}\left(\partial_{z} \phi_{u}\right)^{2}-\frac{2 \epsilon^{2} \eta \partial_{z} \phi_{u}}{1+u} \partial_{x} \phi_{u} \partial_{x} u\right) .
$$

Our approach in $2 \mathrm{~d}$ is similar to the $3 \mathrm{~d}$ case. However the definitions of the function spaces need to be adapted.

Definition 7.1. Given $\kappa \in(0,1)$ we define

$$
W_{q, D}^{2 \alpha}(E):=\left\{\begin{array}{c}
W_{q}^{2 \alpha}(E), 2 \alpha \in[0,1 / q) \\
\left\{u \in W_{q}^{2 \alpha}(E) ; u( \pm 1)=0\right\}, 2 \alpha \in(1 / q, 2]
\end{array}\right.
$$

and

$$
S_{q}(\kappa):=\left\{u \in W_{q, D}^{2}(E) ;\|u\|_{W_{q, D}^{2}(E)}<1 / \kappa,-1+\kappa<u(x) \text { for } x \in I\right\} .
$$

\subsection{Assumptions on the temperature dependence}

As in the $3 \mathrm{~d}$ case we will work with the following, slightly different, hypotheses on the physical parameters:

Definition 7.2. Let $X \subset \mathbb{R}^{3}$ and $Y \subset \mathbb{R}^{2}$ be open and bounded. We say that the 
assumption $\mathrm{C1}(2 \mathrm{~d})$ is satisfied if the following holds:

$$
\left\|\begin{array}{c}
\mu(T) \geq c>0, T \in \mathbb{R}, \mu \in C^{1} \\
\epsilon_{1}: \mathbb{R}^{2} \rightarrow \mathbb{R}, \quad(T, d T) \rightarrow \epsilon_{1}(T, d T) \\
\text { is globally Lipschitz for }(T, d T) \in Y, \\
P: \mathbb{R}^{3} \rightarrow \mathbb{R}, \quad(u, d T, T) \rightarrow P(u, d T, T) \\
\text { is globally Lipschitz for }(u, d T, T) \in X
\end{array}\right\|
$$

We will get better results if we make an additional assumption on the physical parameters which govern the behaviour of the RHS $g$ of equation 7.2 .

Definition 7.3. Let $\kappa \in(0,1), 2 \sigma \in[0,1 / 2), q \in(2, \infty)$ and $\xi \in[0,(q-1) / q)$ be given. Let $g$ be the function defined in (7.4). We say the assumption $\mathrm{C} 2(2 \mathrm{~d})$ is satisfied with $b(\kappa)>0$, if $\epsilon_{1}, \lambda_{1}, \lambda_{2}$ and $P$ are such that there exists $b(\kappa)$ with:

$$
\left\|\begin{array}{c}
\|g(v)\|_{W_{2, D}^{2 \sigma}(E)} \leq b(\kappa), \text { for } v \in S_{q}(\kappa) \\
\|g(v)-g(w)\|_{W_{2, D}^{2 \sigma}(E)} \leq b(\kappa)\|v-w\|_{W_{q, D}^{2-\xi}(E)}, \text { for } v, w \in S_{q}(\kappa)
\end{array}\right\|
$$

\subsection{Well-posedness of the temperature problem}

In order to prove the Lipschitz continuity of the RHS of the equation governing the membrane's deflection, we need the following, to [ELW15, Lemma 2.6] analogous, result:

Theorem 7.4. Let $\xi \in[0,(q-1) / q), \kappa \in(0,1), v \in S_{q}(\kappa)$ and $\alpha \in(\xi, 1)$ be given. There are unique solutions $\phi_{v} \in W_{2}^{2}(\Omega)$ and $T_{v} \in W_{2}^{2}(\Omega)$ to the electro-thermal problem. Furthermore there exists $c(\kappa)>0$ such that:

$$
\begin{aligned}
& \left\|\phi_{v}-\phi_{w}\right\|_{W_{2, D}^{2-\alpha}(\Omega)} \leq c(\kappa)\|v-w\|_{W_{q}^{2-\xi}(E)}, \quad v, w \in S_{q}(\kappa), \\
& \left\|T_{v}-T_{w}\right\|_{W_{q, D}^{2-\alpha}(\Omega)} \leq c(\kappa)\|v-w\|_{W_{q}^{2-\xi}(E)}, \quad v, w \in S_{q}(\kappa) .
\end{aligned}
$$

The proof for the electrostatic potential can be found in [ELW14, Proposition 2.1]. For the temperature we follow a similar approach and have to prove:

Lemma 7.5. Let $q \in(2, \infty)$ and $\kappa \in(0,1)$ be given. For each $v \in S_{q}(\kappa), T_{0} \in C^{\infty}(\Omega)$ 
and $\phi_{u} \in W_{2}^{2}(\Omega)$ there is a unique solution $T_{v} \in W_{2}^{2}(\Omega)$ to

$$
\begin{gathered}
\mathfrak{L}_{u} T_{u}(x, \eta)=-h_{\left(u, \phi_{u}\right)}(x, \eta), \quad(x, \eta) \in \Omega, \\
T_{u}(x, \eta)=T_{0}(x, \eta), \quad(x, \eta) \in \partial \Omega,
\end{gathered}
$$

such that 7.11 is satisfied.

We start by proving the existence of a weak solution for a slightly irregular RHS, which will then allow us to prove a higher regularity for the operator $\mathfrak{L}_{u}$.

Lemma 7.6. For each $v \in S_{q}(\kappa)$ and $F \in W_{2, D}^{-1}(\Omega)$ there is a unique solution $T \in$ $W_{2, D}^{1}(\Omega)$ to the boundary value problem

$$
\begin{gathered}
-\mathfrak{L}_{u} T(x, \eta)=F, \quad(x, \eta) \in \Omega, \\
T(x, \eta)=0, \quad(x, \eta) \in \partial \Omega
\end{gathered}
$$

and there is a constant $c_{2}(\kappa)>0$ such that

$$
\|T\|_{W_{2, D}^{1}(\Omega)} \leq c_{2}(\kappa)\|F\|_{W_{2, D}^{-1}(\Omega)} .
$$

Furthermore, if $F \in L_{2}(\Omega)$, then $T \in W_{2, D}^{2}(\Omega)$ and

$$
\|T\|_{W_{2, D}^{2}} \leq c_{2}(\kappa)\|F\|_{L_{2}}
$$

Proof. According to [Gri11, Def. 1.3.2.3, Eq. $(1,3,2,3)$ ], we may write any $F \in W_{2, D}^{-1}(\Omega)$ in the form $F=f_{0}+\partial_{x} f_{1}+\partial_{\eta} f_{2}$ with $\left(f_{0}, f_{1}, f_{2}\right) \in L_{2}(\Omega)^{3}$. Furthermore $v \in S_{q}(\kappa)$ and $\phi_{v} \in W_{2, D}^{2}(\Omega)$ ensure that $h_{\left(v, \phi_{v}\right)} \in L_{2}(\Omega)$. Therefore we can move $h$ to the RHS and thus GT01, Theorem 8.3] ensures that the boundary value problem has a unique solution $T \in W_{2, D}^{1}(\Omega)$. The two estimates now follow just as in [ELW15, Lemma 2.2].

For $v \in S_{q}(\kappa)$ we define

$$
f_{v}(x, \eta):=\mathfrak{L}_{v} T_{u, 0}+h_{\left(v, \phi_{v}\right)} \in L_{2}(\Omega) .
$$


Now Lemma 7.6 provides us with a unique solution $T_{v} \in W_{2, D}^{2}(\Omega)$ to

$$
\begin{gathered}
-\mathfrak{L}_{u} T_{v}(x, \eta)=f_{v}, \quad(x, \eta) \in \Omega, \\
T_{v}(x, \eta)=0, \quad(x, \eta) \in \partial \Omega .
\end{gathered}
$$

Clearly $S_{v}:=T_{v}+T_{u, 0}$ is a unique solution to 7.12 and $(7.13)$. To prove the Lipschitz dependence of $S_{v}$ on $v \in S_{q}(\kappa)$, we introduce a bounded linear operator

$$
\mathcal{A}(v) \in \mathcal{L}\left(W_{2, D}^{1}(\Omega), W_{2, D}^{-1}(\Omega)\right) \cap \mathcal{L}\left(W_{2, D}^{1}(\Omega), L_{2}(\Omega)\right)
$$

by setting

$$
\mathcal{A}(v) T:=-\mathfrak{L}_{v} T, \quad T \in W_{2, D}^{1}
$$

Note that $\mathcal{A}(v)$ is invertible according to Lemma 7.6 and that $T_{v}=\mathcal{A}(v)^{-1} f_{v}$. For the inverse $\mathcal{A}(v)^{-1}$ we have:

Lemma 7.7. Given $\theta \in[0,1] \backslash\{1 / 2\}$, there is a constant $c_{5}(\kappa)>0$ such that

$$
\left\|\mathcal{A}(v)^{-1}\right\|_{\mathcal{L}\left(W_{2, D}^{\theta-1}(\Omega), W_{2, D}^{\theta+1}(\Omega)\right)} \leq c_{5}(\kappa), v \in S_{q}(\kappa)
$$

The proof is based on the fact that $\mathcal{A}(v)^{-1}$ belongs to both $\mathcal{L}\left(W_{2, D}^{-1}(\Omega), W_{2, D}^{1}(\Omega)\right)$ and $\mathcal{L}\left(L_{2}(\Omega), W_{2, D}^{2}(\Omega)\right)$, which is due to Lemma 7.6. Subsequently it uses complex interpolation. Details can be found in ELW15, Lemma 2.3]. Next we note that $\mathcal{A}(v)$ is Lipschitz continuous with respect to $v$ :

Lemma 7.8. Given $\xi \in[0,(q-1) / q)$ and $\alpha \in(\xi, 1)$, there exists $c_{6}(\kappa)>0$ such that

$$
\|\mathcal{A}(v)-\mathcal{A}(w)\|_{\mathcal{L}\left(W_{2, D}^{2}(\Omega), W_{2, D}^{-\alpha}\right)} \leq c_{6}(\kappa)\|v-w\|_{W_{q}^{2-\xi}(I)}, \quad v, w \in S_{q}(\kappa)
$$

The proof to Lemma 7.8 is identical to the proof of [ELW15, Lemma 2.4] since we consider the same operator.

Now we still need Lipschitz dependence of the RHS. A similar result for an easier case can be found in [ELW15, Lemma 2.5]. 
Lemma 7.9. Given $\xi \in[0,(q-1) / q)$ and $\alpha \in(\xi, 1)$, there exists $c_{7}(\kappa)$ such that:

$$
\left\|f_{v}-f_{w}\right\|_{W_{2, D}^{-\alpha}} \leq c_{7}(\kappa)\|v-w\|_{W_{q}^{2-\xi}, \quad v, w \in S_{q}(\kappa)}
$$

Proof. Because we have a more complex RHS in our temperature equation we will need different estimates than those which can be found in [ELW15, Lemma 2.5]. We use

$$
W_{2}^{1} \cdot W_{2}^{2-\alpha} \hookrightarrow W_{2}^{1 / 2}
$$

and observe that since $v, w \in S_{q}(\kappa)$ and $\phi_{w}, \phi_{v} \in W_{2}^{2}$ there is a $c^{\prime}>0$ and a $c^{\prime \prime}>0$ such that

$$
\begin{aligned}
\left\|h_{v, \phi_{v}}-h_{w, \phi_{w}}\right\|_{W_{2}^{1 / 2}} \leq & c^{\prime}\left(\left\|\partial_{x} \phi_{v}^{2}-\partial_{x} \phi_{w}^{2}\right\|_{W_{2}^{1 / 2}}+\left\|\partial_{z} \phi_{v}^{2}-\partial_{z} \phi_{w}^{2}\right\|_{W_{2}^{1 / 2}}\right. \\
& \left.+\left\|\partial_{x} \phi_{v}\left(\partial_{z} \phi_{v}-\partial_{z} \phi_{w}\right)+\partial_{z} \phi_{w}\left(\partial_{x} \phi_{v}-\partial_{x} \phi_{w}\right)\right\|_{W_{2}^{1 / 2}}\right) \\
\leq & 3 c^{\prime}\left(\left\|\phi_{w}\right\|_{W_{2}^{1}}+\|\left.\phi_{v}\right|_{W_{2}^{1}}\right)\left\|\phi_{v}-\phi_{w}\right\|_{W_{2, D}^{2-\alpha}} \\
\leq & c^{\prime \prime} \| v-\left.w\right|_{W_{q}^{2-\xi}}
\end{aligned}
$$

Using $\mathcal{T}_{0} \in C^{\infty}$ yields

$$
\begin{aligned}
\left|\int_{\Omega}\left(f_{v}-f_{w}\right) k d(x, \eta)\right| & \leq c \int_{\Omega}\left|h_{v, \phi_{v}}-h_{w, \phi_{w}}\right| k d(x, \eta) \\
& \leq c\left\|v-\left.w\right|_{W_{q}^{2-\xi}(I)}|| k\right\|_{W_{2, D}^{\alpha}}
\end{aligned}
$$

In the second estimate we used $v, w \in S_{q}(\kappa), L_{2} \hookrightarrow W_{2, D}^{-\alpha}$ and $W_{2}^{2-\xi} \hookrightarrow W_{\infty}^{1}$ for the first terms. For the last term we used

$$
\begin{aligned}
\int_{\Omega}\left(\left|h_{v, \phi_{v}}-h_{w, \phi_{w}}\right|\right) k d(x, \eta) & \leq\left. c\left\|h_{v, \phi_{v}}-h_{w, \phi_{w}}\right\|\right|_{L_{2}}\|k\|_{W_{2, D}^{\alpha}} \\
& \leq c\|v-w\|_{W_{q}^{2-\xi}(I)}\|k\|_{W_{2, D}^{\alpha}},
\end{aligned}
$$

where the estimate in the last line is due to 7.19 .

We now invoke the Lemmas 7.7, 7.8 and 7.9 in order to obtain the Lipschitz continuity of $S_{v}$ with respect to $v$ : 
Lemma 7.10. Given $\xi \in[0,(q-1) / q)$ and $\alpha \in(\xi, 1)$, there exists $c_{8}(\kappa)$ such that:

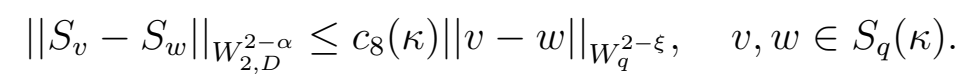

Proof. Let $v, w \in S_{q}(\kappa)$ and recall that $S_{v}=T_{v}+T_{v, 0}$ with $T_{v}=\mathcal{A}(v)^{-1} f_{v} \in W_{2, D}^{2}$. Then obviously $S_{v}-S_{w}=T_{v}-T_{w} \in W_{2, D}^{2-\alpha}$ and so we can split the LHS to obtain

$$
\begin{aligned}
\left\|S_{v}-S_{w}\right\|_{W_{2, D}^{2-\alpha}} \leq & \left\|\mathcal{A}(v)^{-1}\right\|_{\mathcal{L}\left(W_{2, D}^{-\alpha}, W_{2, D}^{2-\alpha}\right)}\left\|f_{v}-f_{w}\right\|_{W_{2, D}^{-\alpha}} \\
+ & \left\|\mathcal{A}(v)^{-1}\right\|_{\mathcal{L}\left(W_{2, D}^{-\alpha}, W_{2, D}^{2-\alpha}\right)}\|\mathcal{A}(v)-\mathcal{A}(w)\|_{\mathcal{L}\left(W_{2, D}^{2}, W_{2, D}^{-\alpha}\right)} \\
& \left\|\mathcal{A}(w)^{-1}\right\|_{\mathcal{L}\left(L_{2}, W_{2, D}^{2}\right)}\left\|f_{w}\right\|_{L_{2}} \\
\leq & c_{8}(\kappa)\|v-w\|_{W_{q}^{2-\xi} .}
\end{aligned}
$$

For the last estimate we applied the estimates (7.16), (7.17) and (7.18).

\subsection{Well-posedness of the deflection problem}

The first step for the proof of the well-posedness result is to analyse the RHS of the equation. See also [ELW15, Prop. 2.1].

Theorem 7.11. Assume that C1(2d) is satisfied. Let $\kappa \in(0,1)$ and $q \in(2, \infty)$ be given and pick $v \in S_{q}(\kappa)$. Then the mapping

$$
\begin{aligned}
g: S_{q}(\kappa) \rightarrow W_{2, D}^{2 \sigma}(E), \quad v \rightarrow & \epsilon_{1}\left(\partial_{\eta} T_{v}(\cdot, 1), T_{v}(\cdot, 1)\right) \lambda_{1}\left(\frac{1+\epsilon^{2}\left|\partial_{x} v\right|^{2}}{(1+v)^{2}}\right)\left|\partial_{\eta} \phi_{v}(\cdot, 1)\right|^{2} \\
& -\lambda_{2} P\left(\partial_{\eta} T_{v}(\cdot, 1), T_{v}(\cdot, 1), v\right)
\end{aligned}
$$

is globally Lipschitz continuous and bounded such that

$$
\|g(v)-g(w)\|_{W_{2, D}^{\nu}(E)} \leq c_{1}\|v-w\|_{W_{q, D}^{2-\xi}(E)}, \text { for } v, w \in S_{q}(\kappa)
$$

with $2 \sigma \in[0,1 / 2), \xi \in[0,1 / 2), \nu \in[0,(1-2 \xi) / 2)$ and $c_{1}(\kappa)>0$.

Proof. In the following we have $2 \sigma \in(\xi+\nu, 1 / 2)$ and $\alpha \in(\xi, 2 \sigma-\nu)$. For equation 
(7.20) we observe that by [ELW14, Eq. (39)] there is $c(\kappa)$ such that

$$
\left\|\partial_{\eta} \phi_{v}(\cdot, 1)\right\|_{W_{2}^{1 / 2}(E)}+\left\|\left|\partial_{\eta} \phi_{v}(\cdot, 1)\right|^{2}\right\|_{W_{2}^{2 \sigma}(E)} \leq c(\kappa)
$$

For $m \in \mathbb{N}$ and $a_{i}, b_{i} \in \mathbb{R}$ the following elementary result $t^{6}$ is evident by induction:

$$
\prod_{i=1}^{m} a_{i}-\prod_{i=1}^{m} b_{i}=\sum_{i=1}^{m}\left(a_{i}-b_{i}\right)\left(\prod_{j=1}^{i-1} b_{j} \prod_{k=i+1}^{m} a_{k}\right)
$$

Applying this with $m=4$ we can split the LHS of 7.20 in the following convenient way:

$$
\begin{aligned}
& \|g(v)-g(w)\|_{W_{2, D}^{\nu}(E)} \\
& \leq \lambda_{1} \|\left(\epsilon_{1}\left(\partial_{\eta} T_{v}(\cdot, 1), T_{v}(\cdot, 1)\right)-\epsilon_{1}\left(\partial_{\eta} T_{w}(\cdot, 1), T_{w}(\cdot, 1)\right)\left(\frac{1+\epsilon^{2}\left|\partial_{x} v\right|^{2}}{(1+v)^{2}}\right)\left|\partial_{\eta} \phi_{v}(\cdot, 1)\right|^{2} \|_{W_{2, D}^{\nu}(E)}\right. \\
& +\lambda_{1} \epsilon^{2}\left\|\left(\left|\partial_{x} v\right|^{2}-\left|\partial_{x} w\right|^{2}\right) \epsilon_{1}\left(\partial_{\eta} T_{w}(\cdot, 1), T_{w}(\cdot, 1)\right)\left(\frac{1+\epsilon^{2}}{(1+v)^{2}}\right)\left|\partial_{\eta} \phi_{v}(\cdot, 1)\right|^{2}\right\|_{W_{2, D}^{\nu}(E)} \\
& +\lambda_{1}\left\|\left(\frac{1}{(1+v)^{2}}-\frac{1}{(1+w)^{2}}\right) \epsilon_{1}\left(\partial_{\eta} T_{w}(\cdot, 1), T_{w}(\cdot, 1)\right)\left(1+\epsilon^{2}\left|\partial_{x} w\right|^{2}\right)\left|\partial_{\eta} \phi_{v}(\cdot, 1)\right|^{2}\right\|_{W_{2, D}^{\nu}(E)} \\
& +\lambda_{1}\left\|\left(\left|\partial_{\eta} \phi_{v}(\cdot, 1)\right|^{2}-\left|\partial_{\eta} \phi_{w}(\cdot, 1)\right|^{2}\right) \epsilon_{1}\left(\partial_{\eta} T_{w}(\cdot, 1), T_{w}(\cdot, 1)\right)\left(\frac{1+\epsilon^{2}\left|\partial_{x} w\right|^{2}}{(1+w)^{2}}\right)\right\|_{W_{2, D}^{\nu}(E)} \\
& +\lambda_{2}\left\|P\left(\partial_{\eta} T_{v}(\cdot, 1), T_{v}(\cdot, 1), v\right)-P\left(\partial_{\eta} T_{w}(\cdot, 1), T_{w}(\cdot, 1), w\right)\right\|_{W_{2, D}^{\nu}(E)} \\
& =: J_{1}+J_{2}+J_{3}+J_{4}+J_{5} .
\end{aligned}
$$

We just have to prove $J_{i} \leq c\|v-w\|_{W_{q, D}^{2-\xi}(E)}$ for $i \in\{1, \ldots, 5\}$. For $J_{5}$ this is simply due to $\mathrm{C} 1(2 \mathrm{~d})$ and Gri11, Theorem 1.5.1.1]. For $J_{2}$ and $J_{3}$ we use [ELW15, Theorem 7.1] (continuity of pointwise multiplication)

$$
W_{q}^{1}(E) \cdot W_{q}^{1-\xi}(E) \cdot W_{2}^{1 / 2}(E) \cdot W_{q}^{2}(E) \cdot W_{2}^{2 \sigma}(E) \hookrightarrow W_{2}^{\nu}(E)
$$

$\overline{{ }^{6} \text { We use the convention } \prod_{i=j}^{k} a_{i}=1 \text { for }} j>k$ and $a_{i} \in \mathbb{R}$. 
To be precise, we estimate:

$$
\begin{aligned}
J_{2} & =\lambda_{1} \epsilon^{2}\left\|\left(\left|\partial_{x} v\right|^{2}-\left|\partial_{x} w\right|^{2}\right) \epsilon_{1}\left(\partial_{\eta} T_{w}(\cdot, 1), T_{w}(\cdot, 1)\right)\left(\frac{1+\epsilon^{2}}{(1+v)^{2}}\right)\left|\partial_{\eta} \phi_{v}(\cdot, 1)\right|^{2}\right\|_{W_{2, D}^{\nu}(E)} \\
& \leq c^{\prime}(\kappa)\left(\left\|\partial_{x} v+\left.\partial_{x} w\right|_{W_{q}^{1}(E)}|| \partial_{x} v-\partial_{x} w\right\|_{W_{q}^{1-\xi}(E)}|| \epsilon_{1}\left(\partial_{\eta} T_{w}(\cdot, 1), T_{w}(\cdot, 1)\right) \|_{W_{2}^{1 / 2}(E)}\right. \\
& \left.\left\|\left(\frac{1+\epsilon^{2}}{(1+v)^{2}}\right)\right\|_{W_{q}^{2}(E)}\left\|\left|\partial_{\eta} \phi_{v}(\cdot, 1)\right|^{2}\right\|_{W_{2}^{2 \sigma}(E)}\right)
\end{aligned}
$$

for $c^{\prime}(\kappa)>0$. For $J_{4}$ we use:

$$
W_{2}^{1 / 2}(E) \cdot W_{q}^{1}(E) \cdot W_{2}^{1 / 2}(E) \cdot W_{2}^{1 / 2-\alpha}(E) \hookrightarrow W_{2}^{\nu}(E)
$$

Having put all the terms in the correct spaces and using $v, w \in S_{q}(\kappa)$, the estimates for $J_{2}$ and $J_{3}$ follow from Theorem 7.4. Finally invoking the continuity

$$
W_{q}^{2}(E) \cdot W_{q}^{1}(E) \cdot W_{q}^{1 / 2}(E) \cdot W_{q}^{1 / 2}(E) \cdot W_{2}^{1 / 2-\alpha}(E) \hookrightarrow W_{2}^{\nu}(E)
$$

we estimate $J_{1}$ :

$$
\begin{aligned}
& \left\|\left(\epsilon_{1}\left(\partial_{\eta} T_{v}(\cdot, 1), T_{v}(\cdot, 1)\right)-\epsilon_{1}\left(\partial_{\eta} T_{w}(\cdot, 1), T_{w}(\cdot, 1)\right)\right)\left(\frac{1+\epsilon^{2}\left|\partial_{x} v\right|^{2}}{(1+v)^{2}}\right)\left|\partial_{\eta} \phi_{v}(\cdot, 1)\right|^{2}\right\|_{W_{2, D}^{\nu}(E)} \\
& \left.\leq c(\kappa) \| \epsilon_{1}\left(\partial_{\eta} T_{v}(\cdot, 1), T_{v}(\cdot, 1)\right)\right)-\epsilon_{1}\left(\partial_{\eta} T_{w}(\cdot, 1), T_{w}(\cdot, 1)\right) \|_{W_{2}^{1 / 2-\alpha}(E)} \\
& \leq c(\kappa)\left\|T_{v}-T_{w}\right\|_{W_{2}^{2-\alpha}(\Omega)} \\
& \leq c(\kappa)\|v-w\|_{W_{q}^{2-\xi}(E)} .
\end{aligned}
$$

The last steps are due to properties of the trace Gri11, Theorem 1.5.1.1], Theorem 7.4 and assumption $\mathrm{C} 1(2 \mathrm{~d})$.

The main result in this section is Theorem 7.12 (see also [ELW15, Theorem 1.1]).

\section{Theorem 7.12.}

Suppose that assumption C1 (2d) is satisfied. Let $q \in(2, \infty)$ be given. For the membrane's displacement consider an initial value $u_{0} \in W_{q, D}^{2}(E)$ such that $u_{0}(x)>-1$ for $x \in E$. Then the following holds true:

1. There is a unique solution $(u, \psi, T)$ to the full system on the maximal interval of 
existence $\left[0, T_{m}\right)$ in the sense that

$$
u \in C^{1}\left(\left[0, T_{m}\right), L_{q}(E)\right) \cap C\left(\left[0, T_{m}\right), W_{q, D}^{2}(E)\right)
$$

satisfies the equations (7.2) and (7.3), while $\psi(t) \in W_{2}^{2}(\Omega(u(t))$ and $T(t) \in$ $W_{2}^{2}(\Omega(u(t))$ (the electro-thermal solutions) solve their corresponding equations in $\Omega(u(t))$ for each $t \in\left[0, T_{m}\right)$.

2. Given $\kappa \in(0,1)$ there is $r(\kappa)>0$ and $b(\kappa)>0$ such that $T_{m}=\infty$, provided that $\left\|u_{0}\right\|_{W_{q}^{2}(E)} \leq r(\kappa)$ and that $C_{2}(2 d)$ is satisfied with $b(\kappa)>0$.

We follow the approach of [ELW15. Let $q \in(2, \infty), \kappa \in(0,1)$ and $\xi \in(0,(q-1) / q)$ be given. Define

$$
Z_{q}(\kappa):=\left\{w \in W_{q}^{2-\xi}(E) ;\|w\|_{W_{q}^{2-\xi}(E)} \leq 1 / \kappa\right\}
$$

and

$$
A(w) v:=-\mu\left(T_{w}\right) \partial_{x}^{2} v=-\mu\left(\mathcal{T}_{0}(\cdot, w(\cdot))\right) \partial_{x}^{2} v
$$

With this notation we can rewrite the equations governing the membrane's deflection as the quasilinear Cauchy problem

$$
\partial_{t} u+A(u) u=-g(u), t>0, u(0)=u_{0}
$$

We start with some basic preparation. While the next Lemma holds true in a much more general setting $]^{7}$ we still take the time to prove it for our specific case.

Lemma 7.13. Consider the linear boundary problem $(\mathcal{A}, \mathcal{B})$ in the sense of [Ama93, 4.]. Furthermore assume that $n=1, a_{11}>0$ and $\mathcal{B} u=\gamma_{\partial} u$ (Dirichlet boundary).

The boundary operator $\mathcal{B}$ satisfies the parameter-dependent Lopatinskii-Shapiro condition (normal complementing condition) with respect to $\mathcal{A}$.

Proof. The principal symbol of the considered linear boundary problem is defined by

$$
a_{\pi}(x, \xi):=a_{11}(x) \xi^{2}, \quad(x, \xi) \in E \times \mathbb{R}
$$

\footnotetext{
${ }^{7}$ For details see for example RR06, Remark 9.44].
} 
and

$$
b_{\pi}(y, \xi):=1, \quad(y, \xi) \in \partial E \times \mathbb{R} .
$$

We denote the tangent bundle of $\partial E$ by $T(\partial E)$. We have to prove that zero is, for each $(x, \xi) \in T(\partial E)$ and $\lambda \in[\operatorname{Re} z \geq 0]$ with $(\xi, \lambda) \neq(0,0)$ the only exponentially decaying solution of the boundary value problem on the half-line:

$$
\begin{gathered}
b_{\pi}\left(x, \xi+\nu(x) i \partial_{t}\right) u(0)=0, \\
{\left[\lambda+a_{\pi}\left(x, \xi+\nu(x) i \partial_{t}\right)\right] u=0, t>0 .}
\end{gathered}
$$

Because we consider a one dimensional space with a Dirichlet boundary, the boundary value problem translates to:

$$
\begin{gathered}
u(0)=0, \\
\left(\lambda+a_{11}(x)\left(\xi+\nu(x) i \partial_{t}\right)^{2}\right) u=0 .
\end{gathered}
$$

Since $\nu(x)$ is the unit-normal this can be reduced to

$$
-a_{11}(x) \partial_{t}^{2} u+\left(\lambda+a_{11}(x) \xi^{2}\right) u=0
$$

The general solution of this ODE is

$$
u(t)=c_{1} e^{t \sqrt{\frac{\lambda}{a_{11}(x)}+\xi^{2}}}+c_{2} e^{-t \sqrt{\frac{\lambda}{a_{11}(x)}+\xi^{2}}}
$$

with $c_{1}, c_{2} \in \mathbb{R}$. Due to 7.27 we get $c_{2}=-c_{1}$. Now $u(t) \stackrel{t \rightarrow \infty}{\longrightarrow} 0$ and $a_{11}>0$ imply $u(t) \equiv 0$.

The following is a generalization of [LLMP04, Prop. 2.4.2]:

Lemma 7.14. Let $2 \leq p<\infty, g \in L_{\infty}(\Omega), \lambda \in \mathbb{C}$ with $\operatorname{Re} \lambda \geq 0$ and $u \in W_{p}^{2}(\Omega) \cap$ $W_{p, D}^{1}(\Omega)$ be given such that $\lambda u-g \Delta u=F \in L_{p}(\Omega)$. Then

$$
\|u\|_{L_{p}} \leq \sqrt{1+\frac{p^{2}}{4}} \frac{1}{c^{2}} \frac{\|F\|_{L_{p}}}{|\lambda|}
$$

holds true, provided that there is a $c>0$ such that $1 / c \leq g(x) \leq c$ for $x \in \Omega$. 
Proof. For $u=0$ the statement is obvious. Now for $u \neq 0$ multiplying 7.31 by $\frac{|u|^{p-2} \bar{u}}{g}$ and integrating yields

$$
\lambda \int_{\Omega}|u|^{p-2} \bar{u} u g^{-1} d x+\int_{\Omega} \sum_{k=1}^{n} \partial_{x_{k}} u \partial_{x_{k}}\left(|u|^{p-2} \bar{u}\right) d x=\int_{\Omega} f|u|^{p-2} \bar{u} g^{-1} d x .
$$

Using the bounds on $g$ and taking the real part we get

$$
R e\left(\lambda c|| u \|_{L_{p}}^{p}+\int_{\Omega} \sum_{k=1}^{n} \partial_{x_{k}} u \partial_{x_{k}}\left(|u|^{p-2} \bar{u}\right) d x\right) \leq \frac{1}{c} \operatorname{Re}\left(\int_{\Omega} f|u|^{p-2} \bar{u} d x\right) .
$$

Now we are in the the same position as in the start of the proof of LLMP04, Prop. 2.4.2] and the result follows by copying the steps that are taken there.

At first we will need some semigroup theory (see also [ELW15, Lemma 3.1]):

Lemma 7.15. Let $q \in(2, \infty), \kappa \in(0,1)$ and $\xi \in(0,(q-1) / q)$ be given. There are $k:=k(\kappa) \geq 1$ and $\omega:=\omega(\kappa)>0$ such that, for each $w \in Z_{q}(\kappa)$,

$$
-2 \omega+A(w) \in \mathcal{H}\left(W_{q, D}^{2}(E), L_{q}(E) ; \kappa, \omega\right)
$$

and $A(w)$ is resolvent positive. Moreover, there is a constant $l(k)>0$ such that

$$
\left\|A\left(w_{1}\right)-A\left(w_{2}\right)\right\|_{\mathcal{L}\left(W_{q, D}^{2}(E), L_{q}(E)\right)} \leq l(\kappa)\left\|w_{1}-w_{2}\right\|_{W_{q}^{2-\xi}(E)}, \quad w_{1}, w_{2} \in Z_{q}(\kappa) .
$$

Proof. Note that due to the continuous embedding $W_{2}^{2}(\Omega) \hookrightarrow L_{\infty}, \mathcal{T}_{0} \in C^{\infty}$ and assumption $\mathrm{C} 1(2 \mathrm{~d})$ there exists $c(\kappa)>0$ such that

$$
1 / c(\kappa)<\mu\left(T_{w}\right)<c(\kappa), w \in Z_{q}(\kappa)
$$

Let $w \in Z_{q}(\kappa)$ be fixed. Since we have estimate $(7.33), A(w)$ is normally elliptic and due to Lemma 7.13 we can use [Ama93, Rem 4.2(c)] to show that $-A(w)$ generates an analytic semigroup on $L_{q}(E)$. Now due to 7.33 we can argue as in ELW15, Lemma 3.1]) to show that the equation

$$
a u-2 \omega u-\mu\left(T_{w}\right) \partial_{x}^{2} u=F
$$


has a unique solution $u \in W_{q, D}^{2}$ for $F \in L_{q}(E), \operatorname{Re} a>0$ and $\omega:=\frac{\pi^{2} c(\kappa)}{2^{3+q / 2}}$. Now Lemma 7.14 yields the resolvent estimate

$$
\left\|(a-2 \omega+A(w))^{-1}\right\|_{\mathcal{L}\left(L_{q}(E)\right)} \leq \frac{c^{\prime}(\kappa)}{|a|}
$$

Finally we can conclude the proof just as in ELW15, Lemma 3.1]) by applying Ama95, I. Rem 1.2.1(a)].

Our operator generates a parabolic evolution operator (see also ELW15, Proposition $3.2])$ :

Lemma 7.16. Let $q \in(2, \infty), \kappa \in(0,1)$ and $\xi \in(0,(q-1) / q)$ be given. Let $\omega(\kappa)>0$ and $l(\kappa)>0$ be as in Lemma 7.15. For $\rho \in(0,1)$ and $N, \tau>0$ given, define

$$
\begin{array}{r}
\mathcal{W}_{\tau}(\kappa):=\left\{w \in C\left([0, \tau], W_{q, D}^{2-\xi}(E)\right) ;\|w(t)-w(s)\|_{W_{q, D}^{2-\xi}(E)} \leq \frac{N}{l(\kappa)}|t-s|^{\rho}\right. \\
\text { and } \left.w(t) \in Z_{q}(\kappa) \text { for } 0 \leq t, s \leq \tau\right\} .
\end{array}
$$

There is a constant $c_{0}(\rho)>0$ independent of $N$ and $\tau$ such that the following holds true: For each $w \in \mathcal{W}_{\tau}(\kappa)$, there exists a unique parabolic evolution operator $U_{A(w)}(t, s)$, $0 \leq s \leq t \leq \tau$, possessing $W_{q, D}^{2}(E)$ as a regularity subspace, and

$$
\left\|U_{A(w)}(t, s)\right\|_{\mathcal{L}\left(W_{q, D}^{2 \alpha}(E), W_{q, D}^{2 \beta}(E)\right)} \leq c_{*}(\kappa)(t-s)^{\alpha-\beta} e^{-\nu(t-s)}, 0 \leq s<t \leq \tau
$$

for $0 \leq \alpha \leq \beta \leq 1$ with $2 \alpha, 2 \beta \neq 1 / q$. Here $c_{*}(\kappa) \geq 1$ depends on $N, \alpha$ and $\beta$ but is independent of $\tau$ and

$$
-\nu:=-\nu(\kappa, \rho, N):=c_{0}(\rho) N^{1 / \rho}-\omega(\kappa)
$$

Moreover, $U_{A(w)}(t, s) \in \mathcal{L}\left(L_{q}(E)\right)$ is a positive operator for $0 \leq s \leq t \leq \tau$.

The proof of Lemma 7.16, which uses Lemma 7.15 and general semigroup results of Ama95 but not the explicit form of $A$ can be found in [ELW15, Proposition 3.2]. We are now ready to prove Theorem 7.12 by applying a fixed point argument in order to find a solution to 7.24 . 
Proof of Theorem 7.12. Let $q \in(2, \infty)$ be given. Let us consider an initial value $u_{0} \in$ $W_{q, D}^{2}(E)$ with $u_{0}(x)>-1$ for $x \in E$. Clearly, there is $\kappa \in(0,1 / 2)$ with

$$
u_{0} \in S_{q}(2 \kappa),\left\|u_{0}\right\|_{W_{q, D}^{2-\xi}(E)} \leq \frac{1}{2 \kappa}
$$

where we fix $\xi$ and $\sigma$ such that:

$$
0<\xi<1 / q, 0<\frac{1}{2}-\frac{1}{q}<2 \sigma<\frac{1}{2}-\xi
$$

Let $4 \rho \in(0, \xi)$ be given. Let $c_{0}(\rho)>0$ be as in Lemma 7.16 and then choose $N>0$ with the property that $-\nu:=c_{0}(\rho) N^{1 / p}-\omega(\kappa)<0$. Since

$$
W_{2, D}^{2 \sigma}(E) \hookrightarrow W_{q, D}^{2 \sigma-\frac{1}{2}+\frac{1}{q}}(E) \hookrightarrow L_{q}(E)
$$

it follows from Lemma 7.16 that, for $w \in \mathcal{W}_{\tau}(\kappa)$ fixed,

$$
\left\|U_{A(w)}(t, s)\right\|_{\mathcal{L}\left(W_{q, D}^{2}(E)\right)}+(t-s)^{-\sigma+1+\frac{1}{2}\left(\frac{1}{2}-\frac{1}{q}\right)}\left\|U_{A(w)}(t, s)\right\|_{\mathcal{L}\left(W_{2, D}^{2 \sigma}(E), W_{q, D}^{2}(E)\right)} \leq c_{*}(\kappa) e^{-\nu(t-s)}
$$

for $0 \leq s \leq t \leq \tau$, where the constant $c_{*}(\kappa)$ is independent of $w$ and $\tau>0$. Now set

$$
\mathcal{V}_{\tau}(\kappa):=\left\{v \in \mathcal{W}_{\tau}(\kappa) ;\|v(t)\|_{W_{q, D}^{2}(E)} \leq 1 / \kappa_{0} \text { and } v(t) \geq-1+\kappa \text { for } 0 \leq t, s \leq \tau\right\}
$$

with $\kappa_{0}:=\kappa / c_{*}(\kappa)$. By Lemma 7.11 there is $c_{1}(\kappa)>0$ s.t.:

$$
\begin{gathered}
\|g(v)-g(w)\|_{W_{2, D}^{2 \sigma}(E)} \leq c_{1}(\kappa)\|v-w\|_{W_{q, D}^{2-\xi}(E)}, \quad v, w \in S_{q}(\kappa) \\
\|g(v)\|_{W_{2, D}^{2 \sigma}(E)} \leq c_{1}(\kappa), v \in S_{q}(\kappa) .
\end{gathered}
$$

We prove that the mapping

$$
\Gamma(v)(t):=U_{A(v)}(t, 0) u_{0}-\int_{0}^{t} U_{A(v)}(t, s) g(v(s)) d s, t \in[0, \tau], v \in V_{\tau}(\kappa),
$$

defines a contraction from $\mathcal{V}_{\tau}(\kappa)$ into itself. Since $U_{A(v)}(t, 0)$ is a positive operator and $u_{0} \geq-1+2 \kappa$, we can apply the embedding $W_{q}^{2}(E) \hookrightarrow L_{\infty}(E)$ with constant 2 and then 
invoke 7.40 and 7.42 to estimate:

$$
\begin{aligned}
\Gamma(v)(t) & \geq-1+2 \kappa-2 \int_{0}^{t}\left\|U_{A(v)}(t, s) g(v(s))\right\|_{W_{q, D}^{2}(E)} d s \\
& \geq-1+2 \kappa-2 c_{*}(\kappa) \int_{0}^{t} e^{-\nu(t-s)}(t-s)^{\sigma-1-\frac{1}{2}\left(\frac{1}{2}-\frac{1}{q}\right)}\|g(v(s))\|_{W_{2, D}^{2 \sigma}(E)} d s \\
& \geq-1+2 \kappa-2 c_{*}(\kappa) c_{1}(\kappa) \int_{0}^{\tau} e^{-\nu s} s^{\sigma-1-\frac{1}{2}\left(\frac{1}{2}-\frac{1}{q}\right)} d s .
\end{aligned}
$$

Furthermore, by the same arguments as in [ELW15, Proof Theorem 1.1] we obtain the remaining four of the five essential estimates

$$
\begin{aligned}
& \|\Gamma(v)(t)\|_{W_{q, D}^{2}(E)} \leq \frac{c_{*}(\kappa)}{2 \kappa}+c_{*}(\kappa) c_{1}(\kappa) \int_{0}^{\tau} e^{-\nu s} s^{\sigma-1-\frac{1}{2}\left(\frac{1}{2}-\frac{1}{q}\right)} d s, \\
& \|\Gamma(v)(t)-\Gamma(w)(t)\|_{W_{q, D}^{2-\xi}(E)} \leq c(\kappa)\left(( \operatorname { m a x } _ { 0 \leq r \leq \tau } r ^ { \frac { \xi } { 2 } } e ^ { - \nu r } ) \left[\left\|u_{0}\right\|_{W_{q, D}^{2}(E)}\right.\right. \\
& \left.\left.+c_{1}(\kappa)\right]+c_{1}(\kappa)\left(\max _{0 \leq r \leq \tau} r^{\xi / 2+\sigma-\frac{1}{2}\left(\frac{1}{2}-\frac{1}{q}\right)}\right)\right)\|v-w\|_{\mathcal{V}_{\tau}(\kappa)},
\end{aligned}
$$

with $c(\kappa)>0$,

$$
\|\Gamma(v)(t)-\Gamma(v)(s)\|_{W_{q, D}^{2-\xi}} \leq m_{*}(\kappa)\left(\max _{0 \leq r \leq \tau} r^{\rho} e^{-\nu r}\right)\left(\left\|u_{0}\right\|_{W_{q, D}^{2-\xi+4 \rho}(E)}+c_{1}(\kappa)\right)(t-s)^{\rho}
$$

with $m_{*}(\kappa)>0$ and

$$
\|\Gamma(v)(t)\|_{W_{q, D}^{2-\xi}(E)} \leq m_{*}(\kappa)\left(\max _{0 \leq r \leq \tau} r^{2 \rho} e^{-\nu r}\right)\left(\left\|u_{0}\right\|_{W_{q, D}^{2-\xi+4 \rho}(E)}+c_{1}(\kappa)\right)+\frac{1}{2 \kappa}
$$

Gathering equations 7.43) 7.47 we can see that one may choose $\tau:=\tau(\kappa)$ sufficiently small such that the mapping $\Gamma: \mathcal{V}_{\tau}(\kappa) \rightarrow \mathcal{V}_{\tau}(\kappa)$ defines a contraction and thus has a unique fixed point $u \in \mathcal{V}_{\tau}$. Therefore the proof of Theorem 7.12 can be concluded just as in [ELW15, Proof Theorem 1.1].

For part two of Theorem 7.12 observe that if $\mathrm{C} 2(2 \mathrm{~d})$ is satisfied with $b(\kappa)>0$, one can replace $c_{1}(\kappa)$ with $b(\kappa)$ in the five estimates. Therefore even for $\tau>0$ arbitrarily large it suffices that $\left\|u_{0}\right\|_{W_{2, D}^{2}} \leq r(\kappa)$ and $b(\kappa)$ sufficiently small for $\Gamma$ to define a contraction. 


\section{Numerical analysis}

In the previous chapters we derived and analysed a highly versatile model which depends on a variety of constants and functions. After these theoretical results we are now interested in the qualitative aspects of the solution. In particular we want to examine the differences between the full model with temperature and the model with just electrostatics. We will now investigate the system numerically, focusing on the membrane's deflection.

\subsection{Numerical setting}

We will stay in the $2 \mathrm{~d}$ case and work on the system in section 7 while using the notation therein. Our approach is based on the numerical analysis in [Lie16: We use the finiteelement method and determine the membrane's displacement via the Crank-Nicolson method. We discretize the space $\Omega$ into the same simple rectangles and the time in intervals. Therefore the variational formulation for the electrostatic problem is equal to the one in [Lie16, 6.1]. For the temperature we change the boundary values and add the right-hand side $h_{u, \psi}$ which now depends on the membrane's deflection and the electrostatic potential. The variational formulation for the deflection is

$$
\begin{aligned}
\left(\partial_{t} u(t), v\right)_{L_{2}(E)}+a_{T}(u(t), v) & =(g(u(t), \psi, T), v)_{L_{2}(E)}, \\
u(0) & =u_{0}
\end{aligned}
$$

with the bilinear form

$$
a_{T}(u, v):=\int_{E} \mu(T) \partial_{x} u \partial_{x} v-\partial_{x} T \partial \mu \partial_{x} u v d x
$$

now depending on the temperature. Due to this new situation we have to implement some changes and in particular use a different numerical scheme: In our setting we have to recalculate the stiffness matrix of the membrane's displacement in every time step because of the dependence on the temperature. Furthermore we obviously add the computation of the temperature after the electrostatics and before the computation of the membrane's new right-hand side in every iteration. 


\subsection{Results}

The previous theoretical analysis is of course valid for all possible combinations which satisfy the necessary conditions. However in order to show some solutions we will need to fix a specific example. Afterwards we can examine the influences of the parameters.

We assume that the cylinder is filled with air at $20^{\circ} \mathrm{C}$ and normal pressure of $10^{5}$ pascal and take the silicone elastomer Sylgard 184 as material for the membrane. We use the ideal gas law inside the cylinder and let $\epsilon_{1}$ be independent of temperature (just for simplicity). This results in the following choices for the full set of parameters which we will call M1:

Width $a=1 \mathrm{~mm}$,

Height $h=0.5 \mathrm{~mm}$,

Applied Voltage (Volt) $V=2$,

Temperature on Boundary $T_{0}=293 K$,

Thermal conductivity $k=26 \frac{\mathrm{W}}{\mathrm{mmK}}$,

Electric conductivity $\sigma=10^{-9} \frac{\mathrm{S}}{\mathrm{mm}}$,

Gas Constant $R=8.314510^{6} \mathrm{~kg} \frac{\mathrm{mm}^{2}}{\mathrm{~s}^{2} \mathrm{molK}}$,

Amount of substance $N=1.641110^{-11} \mathrm{~mol}$,

Shear modulus constants $\mu_{1}=0.373, \mu_{0}=0.0032$,

Permittivity $\epsilon_{1}=11.68$.

These choices result in the following values for the constants and functions used in our PDEs:

$$
\begin{aligned}
& \mu(T)=0.373+0.0032 T, \\
& \lambda_{1}=16, \\
& \lambda_{2}=2, \\
& \lambda_{4}=0.104,
\end{aligned}
$$


$\epsilon=0.5$.

It remains to remark that these choices are not only consistent with our initial modelling in section 4.5 but also with the theoretical analysis in section 7 .

Remark 8.1. Given $N, R>0, q>2$ and $\kappa \in(0,1)$, let $u \in S_{q}(\kappa)$. The ideal gas law given by $P: W_{2, D}^{2-\xi}(E) \rightarrow W_{2, D}^{\nu}(E)$ with

$$
P(u):=\frac{N R T_{u}(\cdot, 1)}{V_{C}(u)}=\frac{N R T_{u}(\cdot, 1)}{2+\int_{-1}^{1} u d x}
$$

is Lipschitz continuous.

Proof. We have to prove that the pressure term is Lipschitz with respect to the membrane's displacement. Let $q>2, \xi \in[0,(q-1) / q), \kappa \in(0,1), u, v \in S_{q}(\kappa)$ and $\nu \in[0,(1-2 \xi) / 2)$ be given. There exists $c>0$ such that:

$$
\begin{aligned}
& \|P(u)-P(v)\|_{W_{2, D}^{\nu}(E)}=\left\|\frac{N R T_{u}(\cdot, 1)}{2+\int_{-1}^{1} u d x}-\frac{N R T_{v}(\cdot, 1)}{2+\int_{-1}^{1} v d x}\right\|_{W_{2, D}^{\nu}(E)} \\
& \leq N R\left\|\frac{2 T_{u}(\cdot, 1)+T_{u}(\cdot, 1) \int_{-1}^{1} v d x-2 T_{v}(\cdot, 1)-T_{v}(\cdot, 1) \int_{-1}^{1} u d x}{4+2 \int_{-1}^{1}(u+v) d x+\left(\int_{-1}^{1} u d x\right)\left(\int_{-1}^{1} v d x\right)}\right\|_{W_{2, D}^{\nu}(E)} \\
& \leq \frac{N R}{\kappa^{2}}\left\|2 T_{u}(\cdot, 1)+T_{u}(\cdot, 1) \int_{-1}^{1} v d x-2 T_{v}(\cdot, 1)-T_{v}(\cdot, 1) \int_{-1}^{1} u d x\right\|_{W_{2, D}^{\nu}(E)} \\
& \leq \frac{2 N R}{\kappa^{2}}\left\|T_{u}(\cdot, 1)-T_{v}(\cdot, 1)\right\|_{W_{2, D}^{\nu}(E)}+\frac{N R}{\kappa^{2}}\left(\left\|T_{u}(\cdot, 1) \int_{-1}^{1}(u-v) d x\right\|_{W_{2, D}^{\nu}(E)}\right. \\
& \left.+\left\|\left(\int_{-1}^{1} u d x\right)\left(T_{u}(\cdot, 1)-T_{v}(\cdot, 1)\right)\right\|_{W_{2, D}^{\nu}(E)}\right) \\
& \leq c\|u-v\|_{W_{2, D}^{2-\xi}(E)} .
\end{aligned}
$$

In this estimate we used $u, v \in S_{q}(\kappa), W_{2, D}^{2-\xi}(E) \hookrightarrow W_{2, D}^{\nu}(E)$ and lastly Theorem 7.4 .

For a numeric solution of the membrane's deflection $u$ we define

$$
u_{\min }:=\min _{x \in[-1,1]} u(x) \text { and } u_{\max }:=\max _{x \in[-1,1]} u(x) .
$$


The overall dynamics of the equation governing the membrane's deflection are pretty straightforward: Whether the membrane is moving up or down is decided by the sign of the right-hand side and therefore depends on whether electrostatic effects pulling the membrane downward or thermal effects pushing the membrane upwards dominate one another. The right-hand side is

$$
g(u, T, \psi):=\underbrace{\epsilon_{1} \lambda_{1} \frac{1+\epsilon^{2}\left|\partial_{x} u\right|^{2}}{(1+u)^{2}}\left|\partial_{\eta} \psi(\cdot, 1)\right|^{2}}_{\text {electrostatic }}-\underbrace{\lambda_{2} \frac{N R T}{2+\int_{-1}^{1} u}}_{\text {thermal }} .
$$

It is therefore no surprise that by computing this model we can't observe strong temperature effects because in the above setting the electrostatic effect's strength $\epsilon_{1} \lambda_{1}=186.68$ is several orders of magnitude greater than the thermal effect's strength $\lambda_{2} N R=0.08$. This adds to the hypothesis that one can, at least in certain situations, disregard temperature when modelling MEMS.

However M1 was only one of the possible combinations of the above parameters. For example it is possible to increase the amount of substance in the cylinder $N$, that is to say to start with a higher initial pressure like in a gas tank. One could also fill the cylinder with a non ideal gas that has better adiabatic attributes and use the van der Waals law for the pressure. Furthermore applying a lower voltage or taking a different material with less permittivity for the membrane will decrease the strength of the electrostatic effects. Lastly it is possible to change the aspect ratio of the cylinder in favour of height because $\lambda_{1}$ scales in that respect with $1 / h^{3}$ and $\lambda_{2}$ only with $1 / h$. Making one or several of these modifications we can easily build a realistic scenario in which thermal effects heavily dominate the electrostatic ones.

Instead of discussing several of these scenarios in detail, we follow the approach of Lie16 and simply fix the electrostatic factor to an explicit value of 1 :

$$
\epsilon_{1} \lambda_{1}=1
$$

In order to get a regime in which neither effect heavily dominates the other one we also 
fix the thermal factor $\lambda_{2} N R=0.005$. We call this setting M2. In order to see that this choice is reasonable one has to keep in mind that the temperature is measured in Kelvin. Thus the thermal- and electrostatic effect's strengths will be of the same order of magnitude. This simplification makes it possible to discuss several interesting effects that cannot be seen in the model which neglects temperature effects. Also this allows us to show how each of the parameters affect the membrane's movement.

\subsection{Influences of the parameters}

In the following we will vary a single parameter of M2 and show its effects on the membrane's displacement.

\subsubsection{Temperature}

We start by observing the effects of a change in temperature.

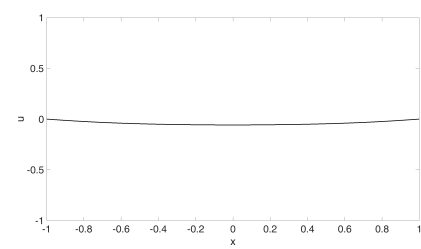

(a) $T_{0}=20^{\circ} \mathrm{C}$ $u_{\min }=-0.0568$

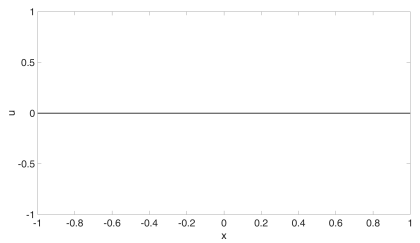

(b) $T_{0}=125^{\circ} \mathrm{C}$

$u_{\min }=-0.001$

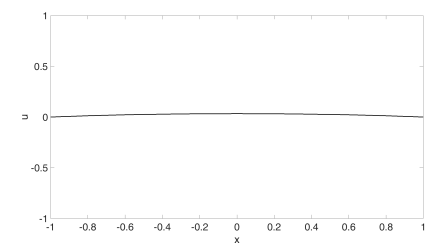

(c) $T_{0}=200^{\circ} \mathrm{C}$ $u_{\max }=0.0331$

Figure 5: M2 with varying temperature at $t=0.4$.

In figure 5 we can see that while under room temperature the membrane still deflects downward, there is a temperature under which electrostatic and thermal effects cancel out each other, so that the membrane stays in place. This implies that by applying a temperature we can prevent that the membrane touches the ground plate. 8 Also by further increasing the temperature the membrane can even deflect upwards. The deflection at different times, that is $t<0.4$ or $t>0.4$, exhibits the same qualitative behaviour.

\footnotetext{
${ }^{8}$ This can be interpreted as the numerical manifestation of the abstract global existence result in part two of Theorem 7.12 .
} 


\subsubsection{Shear modulus}

The shear modulus $\mu(T)$ is a measure for the stiffness of the membrane. We show how decreasing the shear modulus influences the membrane's displacement.

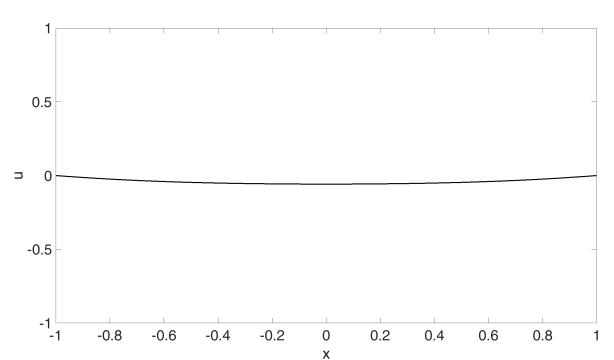

(a) $T_{0}=20^{\circ} \mathrm{C}$ $u_{\text {min }}=-0.0568$

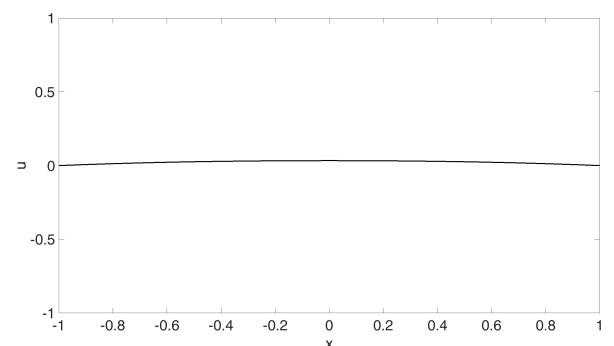

(b) $T_{0}=200^{\circ} \mathrm{C}$ $u_{\max }=0.0331$

Figure 6: M2 with $\mu_{1}=0.373$ at $t=0.4$.

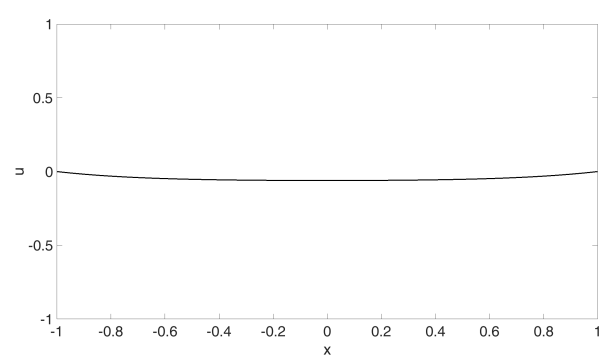

(a) $T_{0}=20^{\circ} \mathrm{C}$ $u_{\text {min }}=-0.0607$

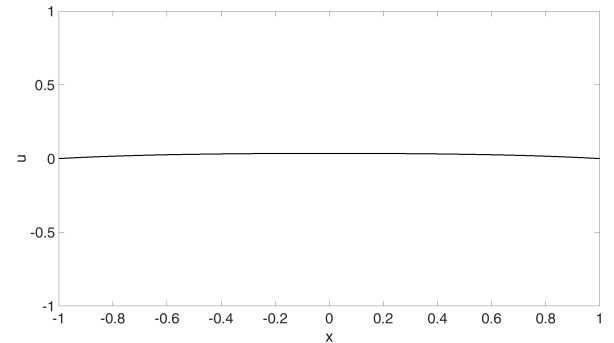

(b) $T_{0}=200^{\circ} \mathrm{C}$ $u_{\max }=0.0352$

Figure 7: M2 with (decreased) $\mu_{1}=0.01$ at $t=0.4$.

By comparing figure 6 with figure 7 we can see that by lowering the shear modulus we make the membrane more bendable and therefore increase the absolute value of the deflection.

\subsubsection{No blowup}

Since we can observe the new effect of the membrane being pushed upwards, we want to examine whether a blowup is likely. 


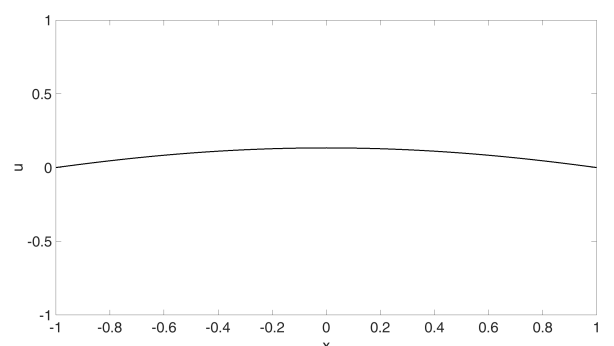

(a) $t=100$,

$u_{\max }=0.1397$

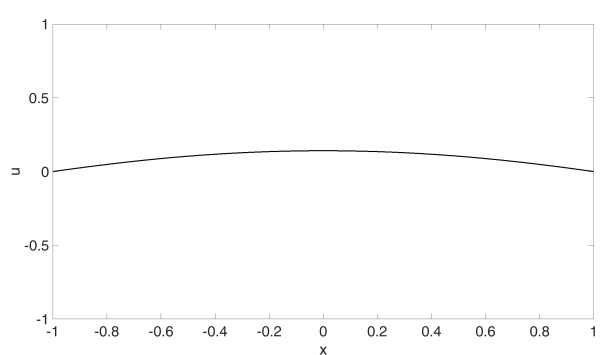

(c) $t=200$,

$u_{\max }=0.1405$

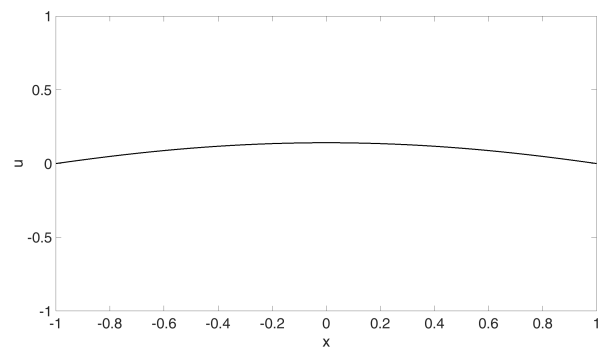

(b) $t=150$,

$u_{\max }=0.1402$

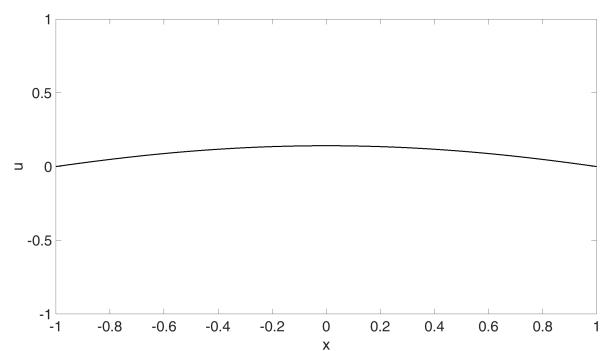

(d) $t=250$,

$u_{\max }=0.1406$

Figure 8: M2 with $T_{0}=200^{\circ} \mathrm{C}$.

Figure 8 gives numerical evidence, that even when thermal effects dominate, the membrane's displacement does not increase indefinitely. Instead the movement stagnates at a certain point dependent on the strength of the thermal effects. This is due to the fact, that when the volume of the cylinder increases, the pressure drops. In the ideal gas law this is evident through the term $\frac{1}{2+\int_{-1}^{1} u}$ which decreases the thermal effect's strength when the membrane's upward deflection increases.

In summary our model captures several new effects that cannot be observed without temperature. We also found that all of the tested parameters influenced the membrane's deflection in exactly the expected way. 


\subsection{Comparison between our model and the one without temperature effects}

Lastly, we want to show a direct comparision between our model with temperature and the model without temperature. The choice of parameters in Lie16 is not physcially motivated, instead all parameters are scaled to 1 . We can fully recover the numerical results of [Lie16] for the case of a constant permittivity profile in our model. In order to do this we disable the temperature effects by simply fixing $\mu(T)=1$ and $\lambda_{2}=0$. Also we fix $\lambda_{1} \epsilon_{1}=1$. This setting is called M0.

\subsubsection{M0 vs M2}

The next figures show the time evolution of the membrane's deflection for the model M0 and our model M2.

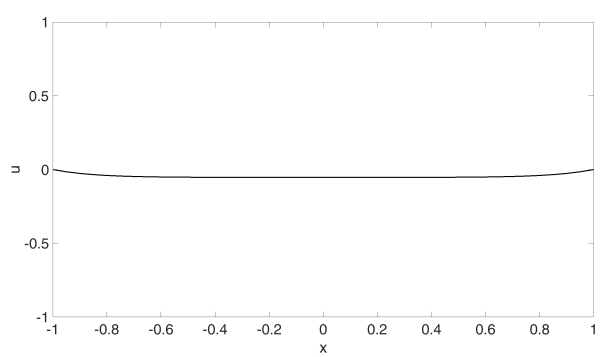

(a) $t=0.1$,

$u_{\text {min }}=-0.0529$

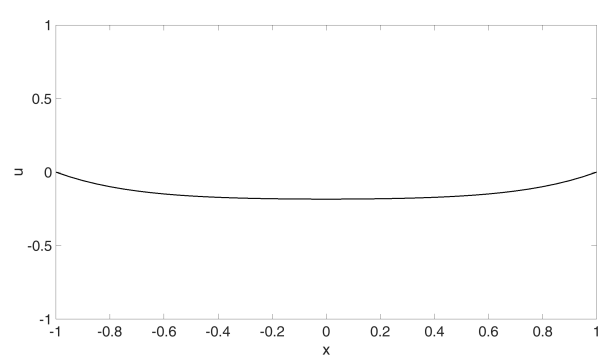

(c) $t=0.3$, $u_{\text {min }}=-0.1837$

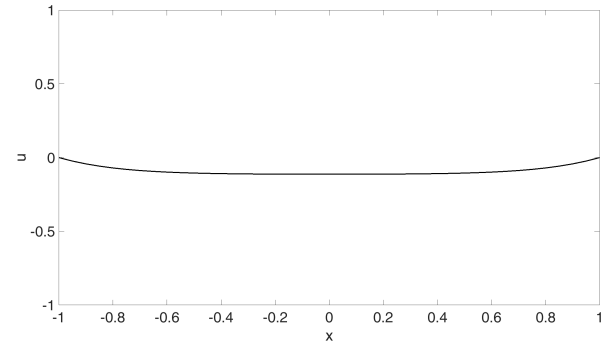

(b) $t=0.2$,

$u_{\text {min }}=-0.1129$

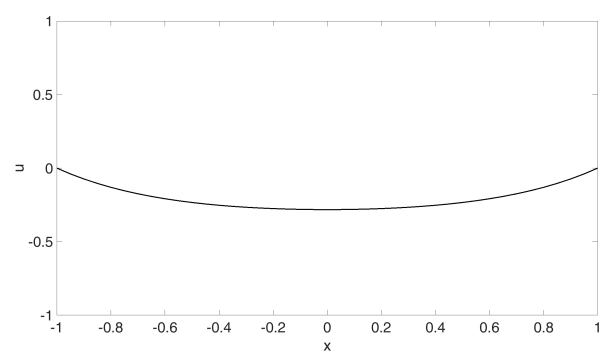

(d) $t=0.4$, $u_{\text {min }}=-0.2822$

Figure 9: Membrane's deflection (M0). 


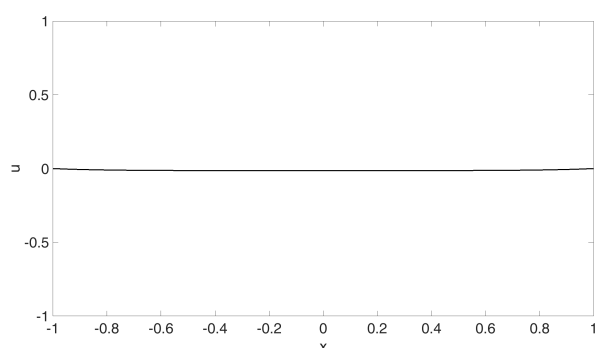

(a) $t=0.1$,

$$
u_{\text {min }}=-0.0138
$$

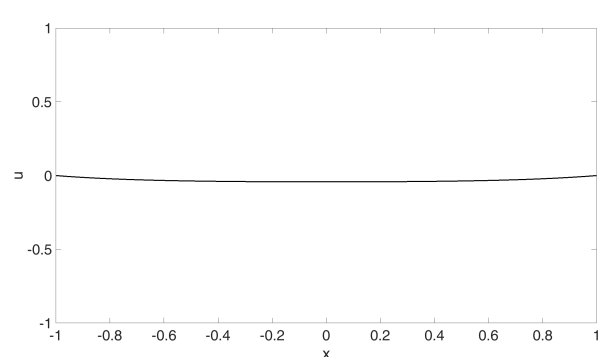

(c) $t=0.3$,

$u_{\text {min }}=-0.0426$

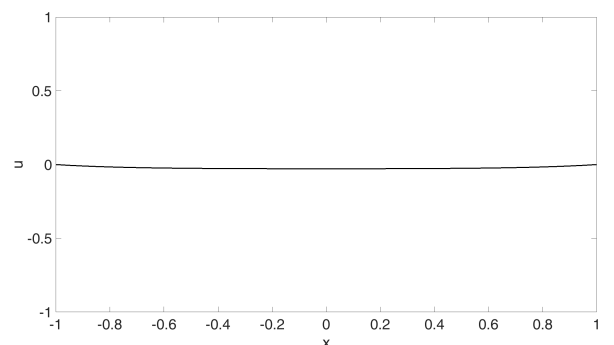

(b) $t=0.2$,

$u_{\text {min }}=-0.0282$

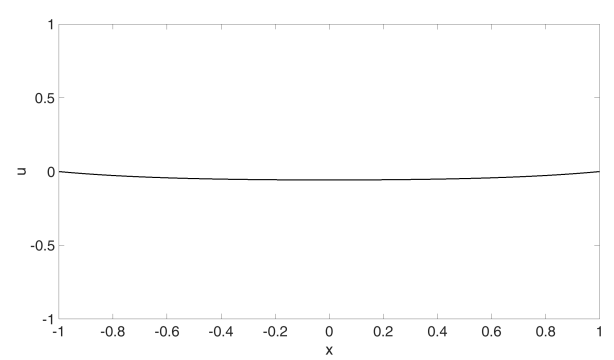

(d) $t=0.4$,

$u_{\text {min }}=-0.0568$

Figure 10: Membrane's deflection (M2) at $20^{\circ} \mathrm{C}$.

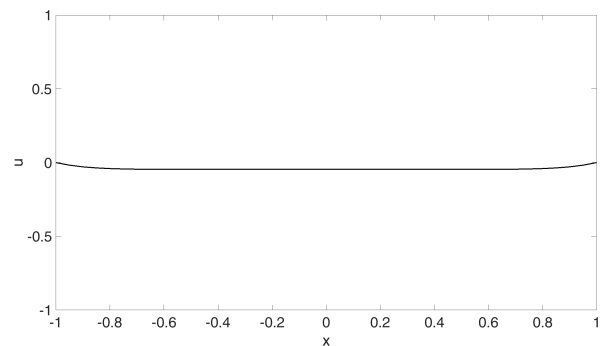

(a) $t=0.1$,

$$
u_{\text {min }}=-0.0461
$$

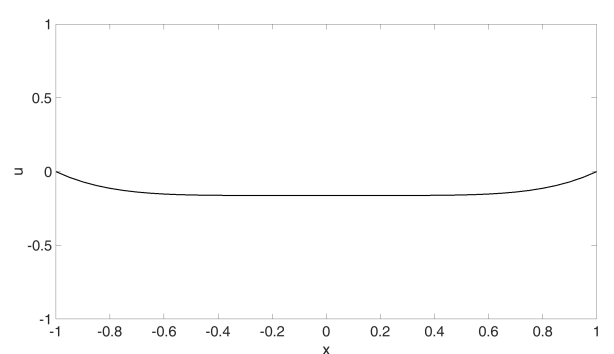

(c) $t=0.3$,

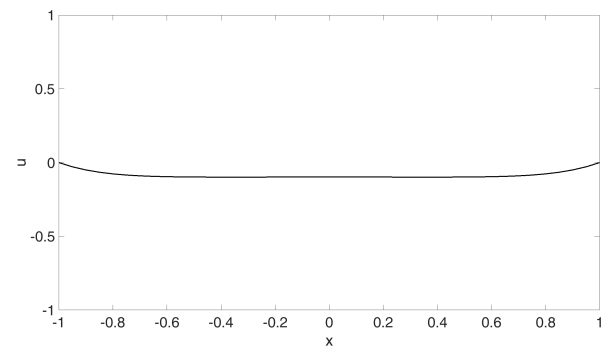

(b) $t=0.2$, $u_{\text {min }}=-0.0984$

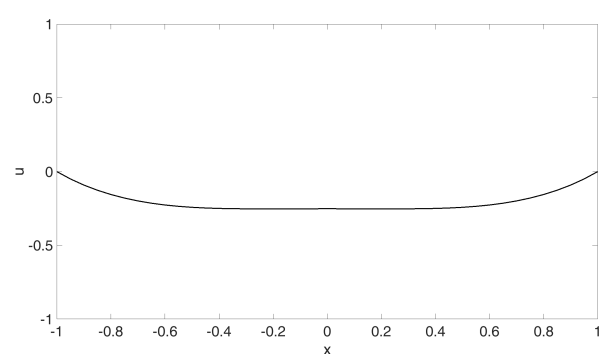

(d) $t=0.4$, $u_{\text {min }}=-0.2528$

Figure 11: Membrane's deflection (M2) at $-220^{\circ} \mathrm{C}$. 
Comparing figure 9 with figure 10 we can see that incorporating temperature changes the membrane's deflection significantly if one assumes a temperature of $20^{\circ} \mathrm{C}$. At $-220^{\circ} \mathrm{C}$ however, the two models behave in a much more similar way.

\subsubsection{M0 vs M2'}

One could argue the outcome of the above comparison is mainly due to the different scaling of the two models. Therefore we will repeat the comparison but this time rescale M2 in similar fashion: We fix the shear modulus constant $\mu_{1}=0.063$. This results in the shear modulus $\mu(T)=0.063+0.0032 T$ and in particular we get $\mu(293)=1$. Thus the shear moduli of our two models are equal at room temperature $\left(20^{\circ} \mathrm{C}\right)$. We let the scaling of the right-hand side of the membrane equation be motivated by our initial physical set of parameters M1: Since M0 uses $\lambda_{1} \epsilon_{1}=1$ instead of $\lambda_{1} \epsilon_{1}=186.68$, we rescale the right-hand side, by the factor $\frac{1}{186.68}$ in order to get $\lambda_{1} \epsilon_{1}=1$ as well. This results in $\lambda_{2} N R=0.0004$ instead of $\lambda_{2} N R=0.08$. We call this setting M2'.

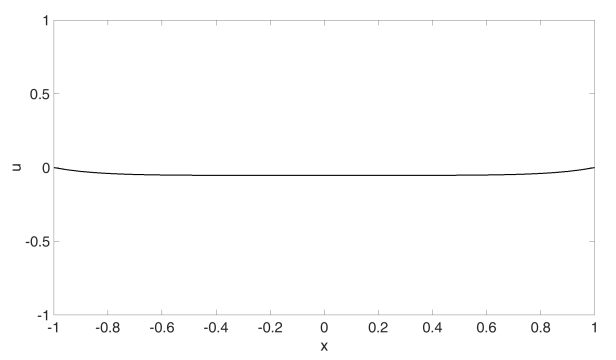

(a) $t=0.1, u_{\min }=-0.0529$

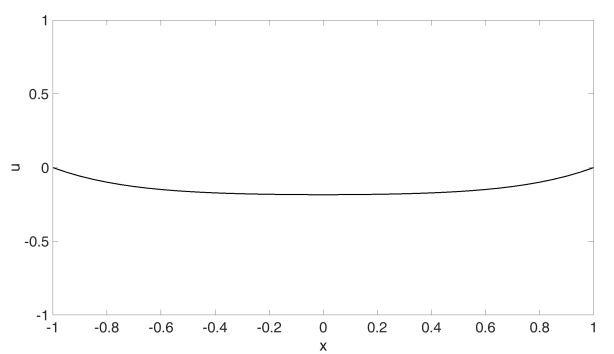

(c) $t=0.3, u_{\min }=-0.1837$

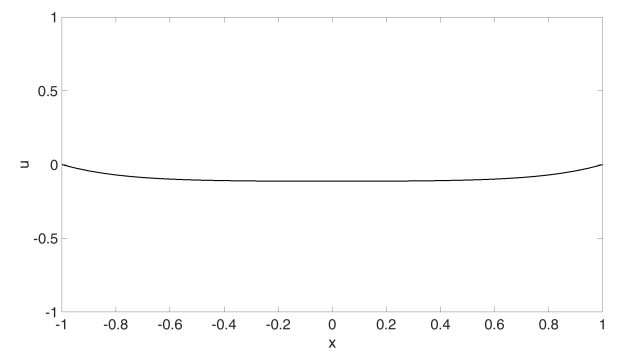

(b) $t=0.2, u_{\min }=-0.1129$

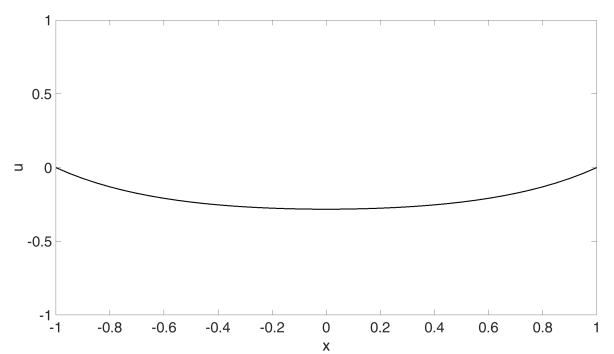

(d) $t=0.4, u_{\min }=-0.2822$

Figure 12: Membrane's deflection (M0). 
8 Numerical analysis

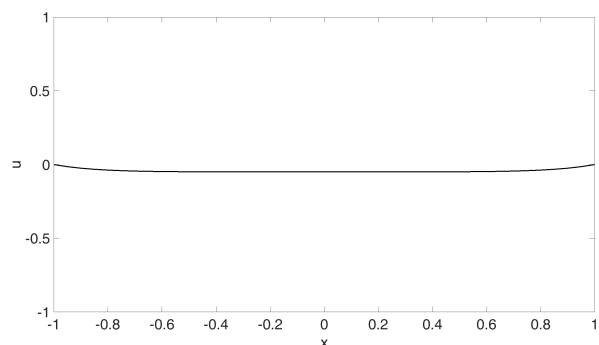

(a) $t=0.1$,

$$
u_{\text {min }}=-0.0497
$$

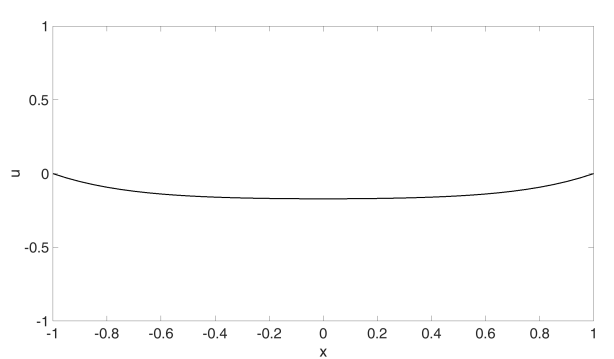

(c) $t=0.3$,

$u_{\text {min }}=-0.171$

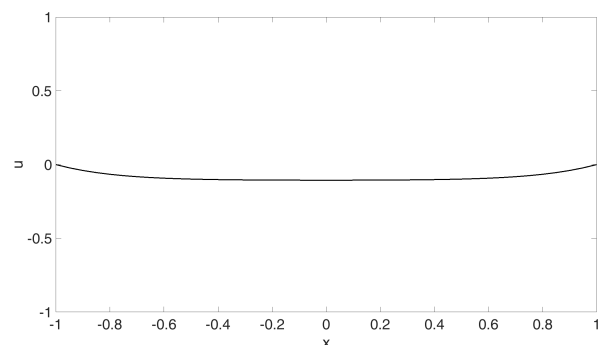

(b) $t=0.2$,

$u_{\text {min }}=-0.1058$

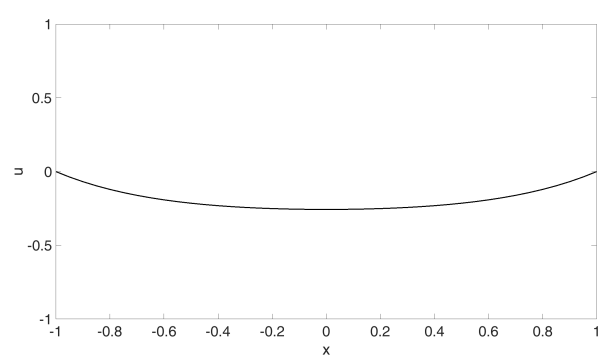

(d) $t=0.4$,

$u_{\text {min }}=-0.2568$

Figure 13: Membrane's deflection (M2') at $20^{\circ} \mathrm{C}$.

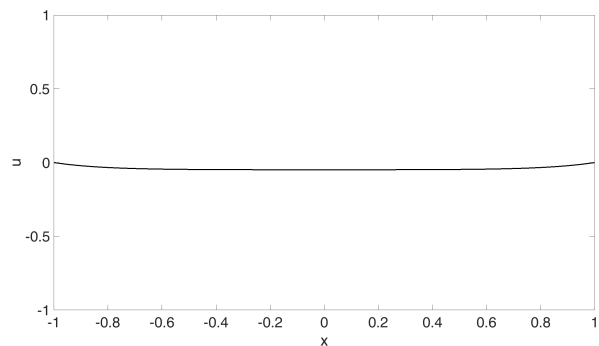

(a) $t=0.1$,

$u_{\text {min }}=-0.0485$

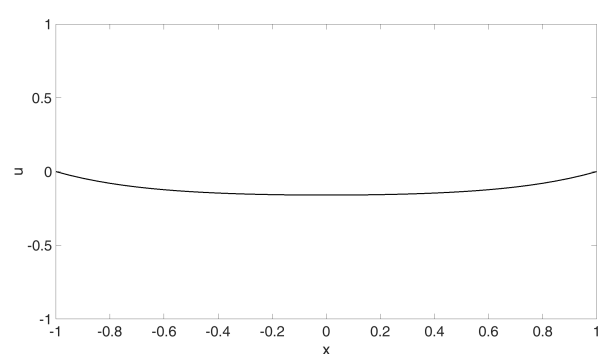

(c) $t=0.3$,

$u_{\text {min }}=-0.1594$

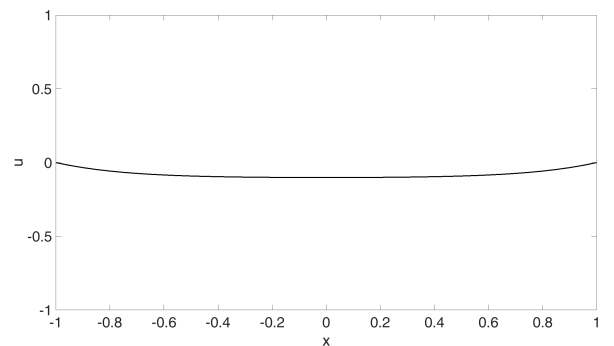

(b) $t=0.2$, $u_{\text {min }}=-0.1015$

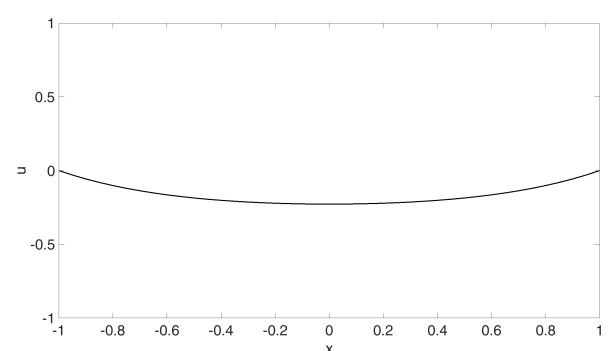

(d) $t=0.4$, $u_{\text {min }}=-0.2274$

Figure 14: Membrane's deflection (M2') at $120^{\circ} \mathrm{C}$. 
Comparing figure 12 with figure 13 we can see that the models behave similar under room temperature. Also, as already mentioned, temperature effects are not dominant in the setting of M2'. Therefore even at $120^{\circ} \mathrm{C}$ we only observe a slight change in behaviour compared to $20^{\circ} \mathrm{C}$. Whereas in the previous comparison in subsection 8.4.1 this increase of $100^{\circ} \mathrm{C}$ changed the membrane's deflection significantly.

We summarize these findings in the following chart which shows the evolution of the deflection's minimum $u_{\text {min }}$ for the different settings:

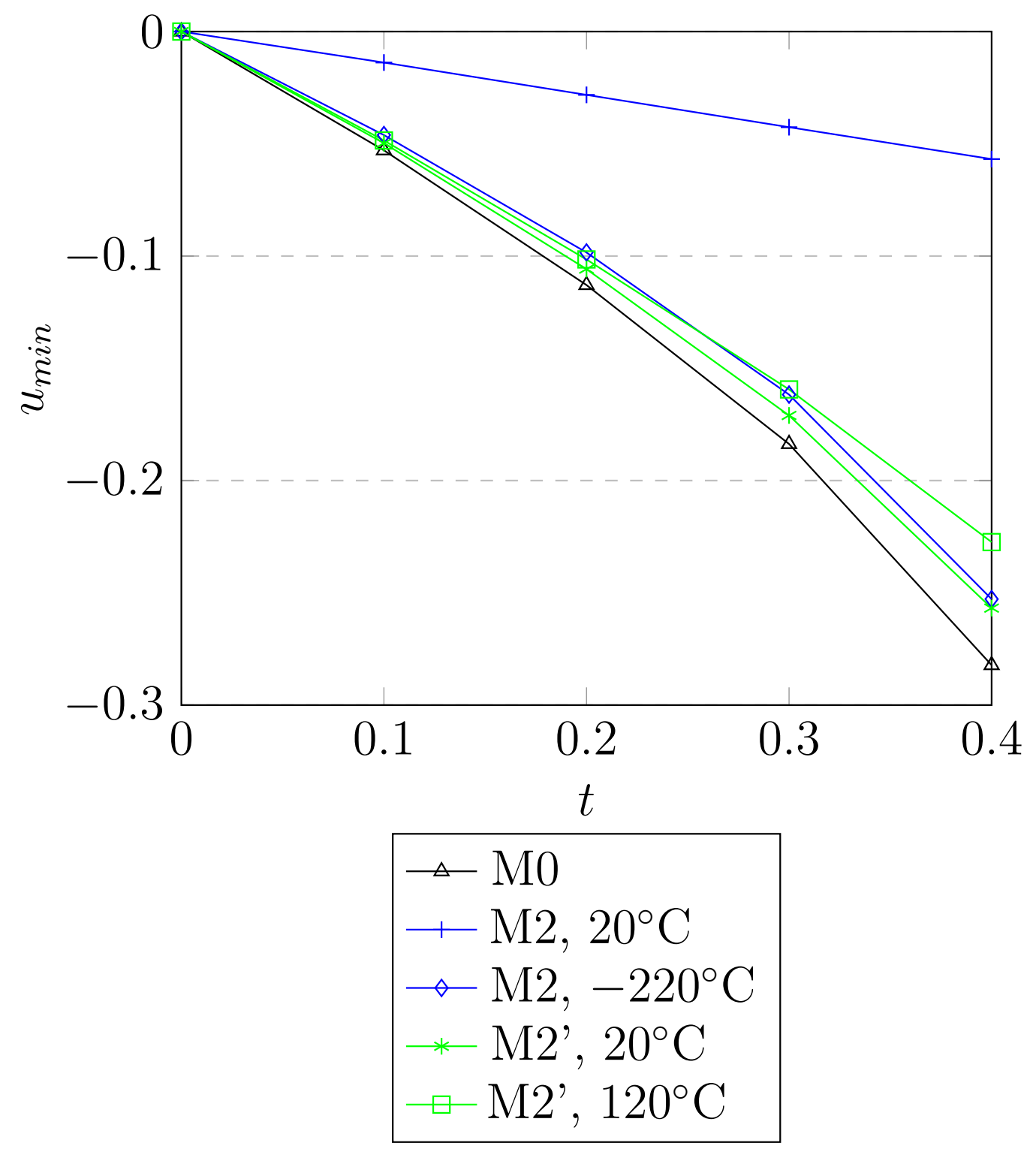




\section{Bibliography}

\section{Bibliography}

[Abi12] R. S. Abiev, Modern state and perspectives of microtechnique application in chemical industry, Russian Journal of General Chemistry 82 (2012), no. 12, 2019-2024.

[AKS17] J. Ahn, K. L. Kuttler, and M. Shillor, Modeling, analysis and simulations of a dynamic thermoviscoelastic rod-beam system, Differential Equations and Dynamical Systems 25 (2017), no. 4, 527-552.

[Ama93] H. Amann, Nonhomogeneous Linear and Quasilinear Elliptic and Parabolic Boundary Value Problems, Function spaces, differential operators and nonlinear analysis, Springer, 1993, pp. 9-126.

[Ama95]_Linear and Quasilinear Parabolic Problems: Volume 1: Abstract Linear Theory, Springer Science \& Business Media, 1995.

[BEL12] M. Bergner, J. Escher, and F. Lippoth, On the blow up scenario for a class of parabolic moving boundary problems, Nonlinear Analysis: Theory, Methods and Applications 75 (2012), no. 10, 3951 - 3963.

[BR98] J. O'M Bockris and A. KN Reddy, Modern Electrochemistry 2B: Electrodics in Chemistry, Engineering, Biology and Environmental Science, vol. 2, Springer Science \& Business Media, 1998.

[Bro03] T. G. Brown, Harsh military environments and microelectromechanical (MEMS) devices, SENSORS, 2003 IEEE, vol. 2, IEEE, 2003, pp. 753-760.

[CA86] D. Cohen and R. Alexander, Chemical reactor theory and problems in diffusion, Physica D: Nonlinear Phenomena 20 (1986), no. 1, 122 - 141.

$\left[\mathrm{EG}^{+} 08\right]$ P. Esposito, N. Ghoussoub, et al., Uniqueness of solutions for an elliptic equation modeling MEMS, Methods and Applications of Analysis 15 (2008), no. $3,341-354$. 


\section{Bibliography}

[EGG10] P. Esposito, N. Ghoussoub, and Y. Guo, Mathematical Analysis of Partial Differential Equations Modeling Electrostatic MEMS, vol. 20, American Mathematical Soc., 2010.

[EL16] J. Escher and C. Lienstromberg, A qualitative analysis of solutions to microelectromechanical systems with curvature and nonlinear permittivity profile, Communications in Partial Differential Equations 41 (2016), no. 1, 134-149.

[EL17]_ _ A survey on second-order free boundary value problems modelling MEMS with general permittivity profile, Discrete \& Continuous Dynamical Systems-Series S 10 (2017), no. 4, 745-771.

[ELW14] J. Escher, P. Laurençot, and C. Walker, A parabolic free boundary problem modeling electrostatic MEMS, Archive for Rational Mechanics and Analysis 211 (2014), no. 2, 389-417.

[ELW15]__ Dynamics of a free boundary problem with curvature modeling electrostatic MEMS, Transactions of the American Mathematical Society 367 (2015), no. 8, 5693-5719.

[EMW18] J. Escher, B. Matioc, and C. Walker, The domain of parabolicity for the muskat problem, Indiana Univ. Math. J. 67 (2018), 679-737.

[FMPS07] G. Flores, G. Mercado, J. A. Pelesko, and N. Smyth, Analysis of the dynamics and touchdown in a model of electrostatic MEMS, SIAM Journal on Applied Mathematics 67 (2007), no. 2, 434-446.

[GG08] N. Ghoussoub and Y. Guo, On the partial differential equations of electrostatic MEMS devices II: Dynamic case, Nonlinear Differential Equations and Applications NoDEA 15 (2008), no. 1-2, 115-145.

[GL02] S. Grossmann and D. Lohse, Prandtl and Rayleigh number dependence of the Reynolds number in turbulent thermal convection, Physical Review E 66 (2002), no. 1, 016305. 


\section{Bibliography}

[GPW05] Y. Guo, Z. Pan, and M. J. Ward, Touchdown and pull-in voltage behavior of a MEMS device with varying dielectric properties, SIAM Journal on Applied Mathematics 66 (2005), no. 1, 309-338.

[Gri85] P. Grisvard, Elliptic Problems in Nonsmooth Domains, Monographs and Studies in Mathematics. Pitman, vol. 24, Advanced Publishing Program, Boston, MA, 1985.

[Gri11]_ _ Elliptic Problems in Nonsmooth Domains, vol. 69, SIAM, 2011.

[GT01] D. Gilbarg and N. S. Trudinger, Elliptic Partial Differential Equations of Second Order, Springer-Verlag Berlin Heidelberg, 2001.

[Guo08] Yujin Guo, Global solutions of singular parabolic equations arising from electrostatic MEMS, Journal of Differential Equations 245 (2008), no. 3, 809844.

[GZZ19] Y. Guo, Y. Zhang, and F. Zhou, Singular behavior of an electrostatic-elastic membrane system with an external pressure, arXiv preprint arXiv:1902.03707 (2019).

[Hui11] K. M. Hui, The existence and dynamic properties of a parabolic nonlocal MEMS equation, Nonlinear Analysis: Theory, Methods and Applications 74 (2011), no. 1, 298-316.

[JMTT14] I. D. Johnston, D. K. McCluskey, C. K. L. Tan, and M. C. Tracey, Mechanical characterization of bulk Sylgard 184 for microfluidics and microengineering, Journal of Micromechanics and Microengineering 24 (2014), no. 3, 035017, $7 \mathrm{pp}$.

[Kaa09] V. Kaajakari, Practical MEMS: Design of Microsystems, Accelerometers, Gyroscopes, RF MEMS, Optical MEMS, and Microfluidic Systems, Small Gear Publishings, 2009.

[KHT16] E. K. Kakhki, S. M. Hosseini, and M. Tahani, An analytical solution for thermoelastic damping in a micro-beam based on generalized theory of thermoe- 


\section{Bibliography}

lasticity and modified couple stress theory, Applied Mathematical Modelling 40 (2016), no. 4, 3164-3174.

$\left[\mathrm{KPK}^{+} 14\right]$ U. Kvell, M. Puusepp, F. Kaminski, J. Past, K. Palmer, T. Grönland, and M. Noorma, Nanosatellite orbit control using MEMS cold gas thrusters, Proceedings of the Estonian Academy of Sciences 63 (2014), no. 2, 279.

[Lie15] C. Lienstromberg, A free boundary value problem modelling microelectromechanical systems with general permittivity, Nonlinear Analysis: Real World Applications 25 (2015), 190-218.

[Lie16]__ On qualitative properties of solutions to microelectromechanical systems with general permittivity, Monatshefte für Mathematik 179 (2016), no. $4,581-602$.

[LLMP04] L. Lorenzi, A. Lunardi, G. Metafune, and D. Pallara, Analytic semigroups and reaction-diffusion problems, Internet Seminar, vol. 2005, 2004, p. 127.

[LU73] O. A. Ladyzhenskaya and N. Ural'tseva, Linear and Quasilinear Equations of Elliptic Type.

[LW13] P. Laurençot and C. Walker, A stationary free boundary problem modeling electrostatic MEMS, Archive for Rational Mechanics and Analysis 207 (2013), no. 1, 139-158.

[LW14a] _ A fourth-order model for MEMS with clamped boundary conditions, Proceedings of the London Mathematical Society 109 (2014), no. 6, 14351464.

[LW14b] _ A free boundary problem modeling electrostatic MEMS: I. Linear bending effects, Mathematische Annalen 360 (2014), no. 1-2, 307-349.

[LW14c] _ _ A free boundary problem modeling electrostatic MEMS: II. Nonlinear bending effects, Mathematical Models and Methods in Applied Sciences 24 (2014), no. 13, 2549-2568. 


\section{Bibliography}

[LW16]_ On a three-dimensional free boundary problem modeling electrostatic MEMS, Interfaces and Free Boundaries 18 (2016), no. 3, 393-411.

[LW17a] P. Laurencot and C. Walker, A constrained model for MEMS with varying dielectric properties, Journal of Elliptic and Parabolic Equations 3 (2017), no. $1-2,15-51$.

[LW17b] P. Laurençot and C. Walker, Some singular equations modeling MEMS, Bulletin of the American Mathematical Society 54 (2017), no. 3, 437-479.

[NAMT08] A. Nisar, N. Afzulpurkar, B. Mahaisavariya, and A. Tuantranont, MEMSbased micropumps in drug delivery and biomedical applications, Sensors and Actuators B: Chemical 130 (2008), no. 2, 917-942.

[Paz12] A. Pazy, Semigroups of Linear Operators and Applications to Partial Differential Equations, vol. 44, Springer Science \& Business Media, 2012.

[PB02] J. A. Pelesko and D. H. Bernstein, Modeling MEMS and NEMS, CRC press, 2002.

[PR76] D. Peng and D. B. Robinson, A new two-constant equation of state, Industrial \& Engineering Chemistry Fundamentals 15 (1976), no. 1, 59-64.

[RR06] Michael Renardy and Robert C Rogers, An Introduction to Partial Differential Equations, vol. 13, Springer Science \& Business Media, 2006.

[Sin13] R.N. Singh, Advection diffusion equation models in near-surface geophysical and environmental sciences, J. Ind. Geophys. Union 17 (2013), 117-127.

[SKR $\left.{ }^{+} 01\right]$ V. Svorčík, J. Králová, V. Rybka, J. Plešek, J. Červená, and V. Hnatowicz, Temperature dependence of the permittivity of polymer composites, Journal of Polymer Science Part B: Polymer Physics 39 (2001), no. 8, 831-834.

[TL01] W. Tang and A. Lee, Defense applications of MEMS, Mrs Bulletin 26 (2001), no. $4,318-319$. 


\section{Bibliography}

[VGP $\left.{ }^{+} 06\right] \quad$ J. Vila, P. Ginés, J.M. Pico, C. Franjo, E. Jiménez, L.M. Varela, and O. Cabeza, Temperature dependence of the electrical conductivity in emim-based ionic liquids: Evidence of Vogel-Tamman-Fulcher behavior, Fluid Phase Equilibria 242 (2006), no. 2, $141-146$.

[WSXL08] L. Wang, D. Sipe, Y. Xu, and Q. Lin, A MEMS thermal biosensor for metabolic monitoring applications, Journal of microelectromechanical systems 17 (2008), no. 2, 318-327. 


\section{Curriculum Vitae}

Akademischer Werdegang

Seit $10 / 2016$

Mitglied des internationalen Graduiertenkollegs IRTG 1627

Seit $10 / 2016$

Doktorand am Institut für Angewandte Mathematik, Leibniz Universität Hannover

Mathematical Modelling and Analysis of Temperature Effects in $M E M S$

Betreuung: Prof. Dr. Joachim Escher

$09 / 2016$

Master of Science Mathematik, Universität Bielefeld

Quadratische Klein-Gordon-Gleichung mit rauen Anfangsdaten

Betreuung: Prof. Dr. Sebastian Herr

10/2014 Bachelor of Science Mathematik, Universität Bielefeld

Zufällige Spannbäume und Wilson's Algorithmus

Betreuung: Prof. Dr. Holger Kösters

$06 / 2011$

Abitur, Königin-Mathilde-Gymnasium Herford 